\title{
Multihop relaying and multiple antenna techniques: performance trade-offs in cellular systems
}

\author{
Kevin R Jacobson ${ }^{1,2}$ and Witold A Krzymień ${ }^{1,2^{*}}$
}

\begin{abstract}
Two very important and active areas of wireless research are multihop relaying and multiple antenna techniques. Wireless multihop relaying can increase the aggregate network data capacity and improve coverage of cellular systems by reducing path loss, mitigating shadowing, and enabling spatial reuse. In particular, multihop relaying can improve the throughput for mobiles suffering from poor signal to interference and noise ratio at the edge of a cell and reduce cell size to increase spectral efficiency. On the other hand, multiple antenna techniques can take advantage of scattering in the wireless channel to achieve higher capacity on individual links. Multiple antennas can provide impressive capacity gains, but the greatest gains occur in high scattering environments with high signal to interference and noise ratio, which are not typical characteristics of cellular systems. Emerging standards for fourth generation cellular systems include both multihop relaying and multiple antenna techniques, so it is necessary to study how these two work jointly in a realistic cellular system. In this paper, we look at the joint application of these two techniques in a cellular system and analyze the fundamental tradeoff between them. In order to obtain meaningful results, system performance is evaluated using realistic propagation models.
\end{abstract}

Keywords: MIMO transmission, Multiple antennas, Multihop relaying, Cross-layer design, 4G cellular networks, LTEAdvanced

\section{Introduction}

The key goals for future broadband cellular systems are: reliable data transmission up to $1 \mathrm{~Gb} / \mathrm{s}$ at high spectral efficiency, good coverage throughout the cells, and the ability to reliably serve a large number of mobile users. However, the wireless channel is a very difficult communications channel over which to achieve reliable high speed data transmission. Due to numerous impairments, such as multipath propagation, random fading, high signal losses, and interference, a strongly attenuated and corrupted signal appears at the receiver. In order to overcome this problem, wireless systems must use sophisticated transmission and receiver processing techniques in order to achieve satisfactory throughput at an acceptable error rate. Cellular systems are interference limited by design in order to maximize their capacity.

\footnotetext{
* Correspondence: wak@ece.ualberta.ca

'Department of Electrical and Computer Engineering, University of Alberta, Edmonton, AB, T6G 2V4, Canada

Full list of author information is available at the end of the article
}

As a result, mobile users suffer from low signal to interference and noise ratio (SINR), especially when they are at cell edges. This work considers two techniques that hold promise to further improve spectral efficiency of cellular systems while preserving their wide area coverage: multihop $(\mathrm{MH})$ relaying and multiple-input multiple-output (MIMO) antenna techniques.

MIMO transmission can improve capacity within a given bandwidth by taking advantage of the rich scattering in a typical wireless channel [1,2]. MIMO spatial multiplexing uses uncorrelated spatial signatures of signals at the receiver to create a number of spatial channels to greatly increase capacity. This approach requires complex physical layer processing at the transmitter and/or receiver, and in order to approach potential capacity, full knowledge of the channel gains between all pairs of transmit and receive antennas. Under some circumstances, channel state information must be known at both the transmit and receive ends. Multiple antennas also create diversity that may be exploited to increase 
reliability of transmissions. Both of these techniques provide the greatest gains in richly scattering channels described by a Rayleigh model. A Rayleigh channel is a channel in which no direct line of sight (LOS) exists, so all of the transmitted energy is scattered (and highly attenuated as a result) prior to reception. MIMO can provide great capacity gains, but essentially, it requires a poor channel to do so. When scattering in the channel is not sufficient (e.g., in some Ricean channels), multiple antennas at the transmitter can be used for beamforming, in which the transmitted beam is steered toward the intended receiver. MIMO spatial multiplexing provides the greatest capacity gains at high SINR; however, cellular systems typically operate at low SINR, with users at cell edges suffering from the poorest SINR.

Multihop relaying [3-5], on the other hand, strives to mitigate transmission impairments by reducing the path loss between transmitter and receiver with the addition of intermediate wireless relays. With a short link hop, the path loss is greatly reduced, and obstacles can be avoided so that the SINR is increased and random signal fluctuations due to both shadowing and scattering are reduced. Higher link capacities and improved reliability can thus be obtained. With the higher SINR provided by multihop relaying, it is expected that MIMO techniques may perform better.

It has been observed that a cellular capacity wall of $350 \mathrm{Mb} / \mathrm{s} /$ cell [6] is on the horizon. Therefore, it is necessary to use smaller cells in order to achieve a higher spectral efficiency over an area $\left(\mathrm{b} / \mathrm{s} / \mathrm{Hz} / \mathrm{km}^{2}\right)$. One method of achieving this is to divide the larger cell, typically 1 to 2 kilometer in radius, into smaller subcells in which relay stations (RSs) serve mobile stations (MSs) closest to them. Numerous researchers have looked at the various approaches to $\mathrm{MH}$ relaying in cellular systems [7-12]. Two proposals under consideration for 4G IMT-Advanced [13-15]: IEEE 802.16m [16,17] and LTEAdvanced [18,19] will include relaying as options. Clearly, relaying requires more complicated system level algorithms (medium access control- MAC-layer and higher) in order to achieve good results in a network of wireless stations. Also, $\mathrm{MH}$ relaying requires additional system resources (time or frequency slots), and hence the spectral efficiency (measured in $\mathrm{b} / \mathrm{s} / \mathrm{Hz}$ ) may suffer under some conditions. It seems natural to combine MIMO and relaying techniques in order to improve the performance of a cellular system, but it is necessary to determine how well they work together and what tradeoffs exist in combining them. In addition, it is necessary to use a system model that captures the radio frequency (RF) propagation of a typical cellular system accurately.

There exists a theoretical analysis of MH MIMO systems [20]. However, some results in it have been derived under simplifying assumptions, and the complexity of a deployable MH MIMO system makes it difficult to predict its realistic performance. Thus, we have focussed on simulating and calculating system performance using realistic cellular environments, with parameters and models recommended in emerging standards such as 802.16 [21] and established ones of the 3rd generation partnership project (3GPP) [22]. In particular, the measure of success of MIMO combined with $\mathrm{MH}$ relaying depends greatly on the physical environment in which the system operates. We consider typical urban scenarios, at first analyzing a one-dimensional system and then looking at two-dimensional cellular systems with both hexagonal and Manhattan topologies.

Our work studies a cellular system combining decode and forward (DF) MH relaying with multiple antenna techniques with the goal of achieving higher data carrying capacity simultaneously with good system coverage. Much research has emerged recently on $\mathrm{MH}$ relaying and multiple antennas, which means there are a large number of considerations in the design of such a system. Our initial results in that area were presented in conference papers $[23,24]$. This paper provides a more complete description of the system model used, additional more detailed results, their more extensive and much more insightful discussion and resulting conclusions, which may be of great value to cellular system designers.

The remainder of this paper is structured as follows: Section II provides details on the system model used for the MIMO link, a simple one-dimensional MH MIMO network and a two-dimensional cellular MH MIMO network. Section III gives calculated results for numerous scenarios. Section IV provides some detailed discussion of the results and Section V concludes the paper.

\section{System model}

The MH model used in this paper is an extension of the single antenna MH relaying work in [25-27], in which typical cellular topologies and system parameters are used to calculate network throughput achievable using $\mathrm{MH}$ relaying. In the present paper, which presents and extends the research presented in $[23,24]$, we include the benefits of multiple antenna techniques. The model is necessarily complex, taking into account both physical layer (PHY) and medium access control (MAC) layer considerations. A dual slope path loss model with distance and other parameters typical of cellular systems is used. We capture both non-line of sight (NLOS) Rayleigh and line of sight (LOS) Ricean aspects, which are selected as a function of distance.

\section{A PHY layer model 1) MIMO Link}

The standard MIMO model $[1,2,28]$ is used on each hop of the data link. For a given hop, there are $N_{T}$ transmit 
antennas and $N_{R}$ receive antennas, and the channel is described by an $N_{R} \times N_{T}$ matrix $\mathbf{H}$. Elements of $\mathbf{H}$ are modeled by a random variable that captures the stochastic nature of the wireless channel. We wish to model both line of sight (LOS) and non-line of sight (NLOS) conditions, and so, we express the channel matrix (normalized) as a sum of two components [28]:

$$
\mathbf{H}=\sqrt{\frac{K_{r}}{1+K_{r}}} \mathbf{H}_{\mathrm{LOS}}+\sqrt{\frac{1}{1+K_{r}}} \mathbf{H}_{\mathrm{NLOS}}
$$

$\mathbf{H}_{\mathrm{NLOS}}$ is the NLOS (scattered) component, and its elements are Rayleigh distributed with unity variance. $\mathbf{H}_{\mathrm{LOS}}$ is the LOS (specular) component, and its elements are deterministic. For our work, we assume that $\mathbf{H}_{\mathrm{NLOS}}$ is full rank with $r_{\mathrm{NLOS}}=\min \left(N_{T}, N_{R}\right) . \mathbf{H}_{\mathrm{LOS}}$ has maximum rank $r_{\mathrm{LOS}}=\min \left(N_{T}, N_{R}\right)$ but for propagation distances and antenna array sizes typical of practical cellular systems, $\mathbf{H}_{\mathrm{LOS}}$ is rank-deficient and often has rank $r_{\mathrm{LOS}}=1[28,29] . K_{r}$ is the Rice factor: the ratio of power in the specular component to the power in the scattered component. The capacity of a MIMO link is given by (Endnote $\mathrm{A})$.

$$
R_{E P}(\mathbf{H})=\log _{2}\left[\operatorname{det}\left(\mathbf{I}_{N_{R}}+\frac{\rho}{N_{T}} \mathbf{H H}^{H}\right)\right] \quad \mathrm{b} / \mathrm{s} / \mathrm{Hz}
$$

where $\rho$ is the signal to interference and noise ratio (SINR) at the receiver and $\mathbf{I}_{N_{R}}$ is the identity matrix. SINR is determined by a number of system parameters, such as transmit power, antenna gains, receiver thermal noise, and path loss. The capacity is largest if both $\mathbf{H}_{\mathrm{NLOS}}$ and $\mathbf{H}_{\mathrm{LOS}}$ are full rank, but $\mathbf{H}_{\mathrm{LOS}}$ is usually low rank in practical systems. With low rank $\mathbf{H}_{\mathrm{LOS}}$ and high Rice factor, a significant portion of energy will collapse into fewer eigenmodes of $\mathbf{H}$, and thus, the capacity will be reduced. Monte Carlo simulation with a sufficiently large number of samples can be used to find the average capacity of the MIMO link. However, [29] gives very useful expressions for the upper bound on the average mutual information $\mathrm{E}\left[I_{\mathbf{H}}\right]$ of the Ricean MIMO channel. Special case number 1 (Corollary 1 ) in [29] gives the upper bound for the average mutual information $\mathrm{E}\left[I_{\mathbf{H}}\right]$ of a Ricean channel

$$
R(\mathbf{H})=\mathrm{E}\left[I_{\mathrm{H}}\right] \leq \log _{2}\left[\sum_{p=0}^{K}\left(\frac{\rho b^{2}}{N_{T}}\right)^{p} \sum_{j=0}^{p} K_{r}^{j}(L-p+1)_{(p-j)} \times\left(\begin{array}{c}
K-j \\
p-j
\end{array}\right) \operatorname{tr}_{j}(\mathrm{~T})\right]
$$

where $\rho$ is the $\operatorname{SINR}, K=\min \left(N_{R}, N_{T}\right)$, $b=\sqrt{\frac{1}{K_{r}+1}}, L=\max \left(N_{R}, N_{T}\right),(m)_{n}$ is the Pochhammer symbol given by

$$
(m)_{n}=m(m+1) \cdots(m+n+1)
$$

$\mathbf{T}=\mathbf{H}_{\mathrm{LOS}} \mathbf{H}_{\mathrm{LOS}}^{H}$, and $\operatorname{tr}_{j}(\mathbf{T})$ is the $j$ th elementary symmetric function of $\mathbf{T}$ (see [29] and [30]). Special case number 2 (Corollary 2) in [29] is the case of a Ricean channel with rank $1 \mathbf{H}_{\mathrm{LOS}}$

$$
R(\mathrm{H})=\mathrm{E}\left[I_{\mathrm{H}}\right] \leq \log _{2}\left[1+\sum_{p=1}^{K} \sum_{j=0}^{1}\left(\frac{\rho b^{2}}{N_{T}}\right)^{p}\left(K_{T} K L\right)^{j} \times(L-p+1)_{(p-j)}\left(\begin{array}{c}
K-j \\
p-j
\end{array}\right)\right]
$$

\section{2)One-dimensional multihop relaying system}

Consider the one-dimensional linear MH system shown in Figure 1, in which a base station (BS) wishes to transmit data to the mobile station (MS) at the cell edge via a number of relay stations (RSs). The cell radius, $r$, is divided into $n_{\text {hops }}$ hops, whose distances are $r_{k}^{n_{\text {hops }}}, k=1$, $2, \ldots, n_{\text {hops. }}$. To simplify calculations for the one-dimensional case only, we often use equally spaced relays so that $r_{k}^{n_{\text {hops }}}=r / n_{\text {hops }}, k=1,2, \ldots, n_{\text {hops. }}$. In a MH MIMO system, Figure 2 , there are $n_{\text {hops }}$ channel matrices, $\mathbf{H}_{k}^{n_{\text {hops }}}$, $k=1,2, \ldots, n_{\text {hops. }}$. Hop $k$ has $N_{T, k}$ transmit antennas and $N_{R, k}$ receive antennas.

For each hop, $k$, we have the channel matrix

$$
\mathbf{H}_{k}^{n_{\text {hops }}}=\gamma_{k}^{n_{\text {hops }}} .\left[\sqrt{\frac{K_{r, k}^{n_{\text {hops }}}}{1+K_{r, k}^{n_{\text {hops }}}}} \mathbf{H}_{\mathrm{LOS}, k}^{n_{\text {hops }}}+\sqrt{\frac{1}{1+K_{r, k}^{n_{\text {hop }}}}} \mathbf{H}_{\mathrm{NLOS}, k}^{n_{\text {hoos }}}\right]
$$

where $\gamma_{k}^{n_{\text {hops }}}=\gamma\left(r_{k}^{n_{\text {hops }}}\right)$ and $K_{r, k}^{n_{\text {hops }}}=K_{r}\left(r_{k}^{n_{\text {hops }}}\right)$ are areaaveraged path gain and Rice factor for the $k$ th hop, respectively. The path loss model used is based on the Okumura-Hata and Walfish-Ikegami models for urban macrocell and microcell environments, as these are widely adopted by COST231, 3GPP [22], 802.16 [31] and other standards bodies. Since a benefit of MH relaying is the ability to relay around obstacles, we use a dual slope model, which selects non-line of site (NLOS) or line of sight (LOS) path loss as appropriate. $\gamma(x)$ is given by the path loss model (in $\mathrm{dB}$, and extended to a frequency of $5 \mathrm{GHz}$ [32])

$$
P L_{\mathrm{dB}}(x)=-20 \log _{10}[\gamma(x)]=\left\{\begin{array}{l}
42.5+38.0 \log _{10}(x)+\psi_{\mathrm{dB}} b<x<5,000 \mathrm{~m}, \\
38.2+26.0 \log _{10}(x)+\psi_{\mathrm{dB}} 20 \mathrm{~m}<x<b
\end{array}\right.
$$

where $x$ is distance, and $b$ is the distance breakpoint, below which a NLOS path becomes LOS (typically 300 $\mathrm{m}$ in urban areas). A log-normal random variable, $\psi_{\mathrm{dB}}$,

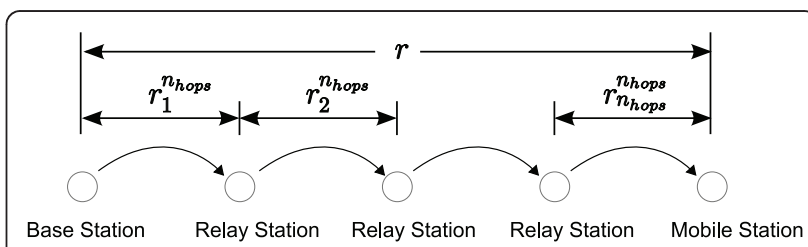

Figure 1 Multihop relaying. 


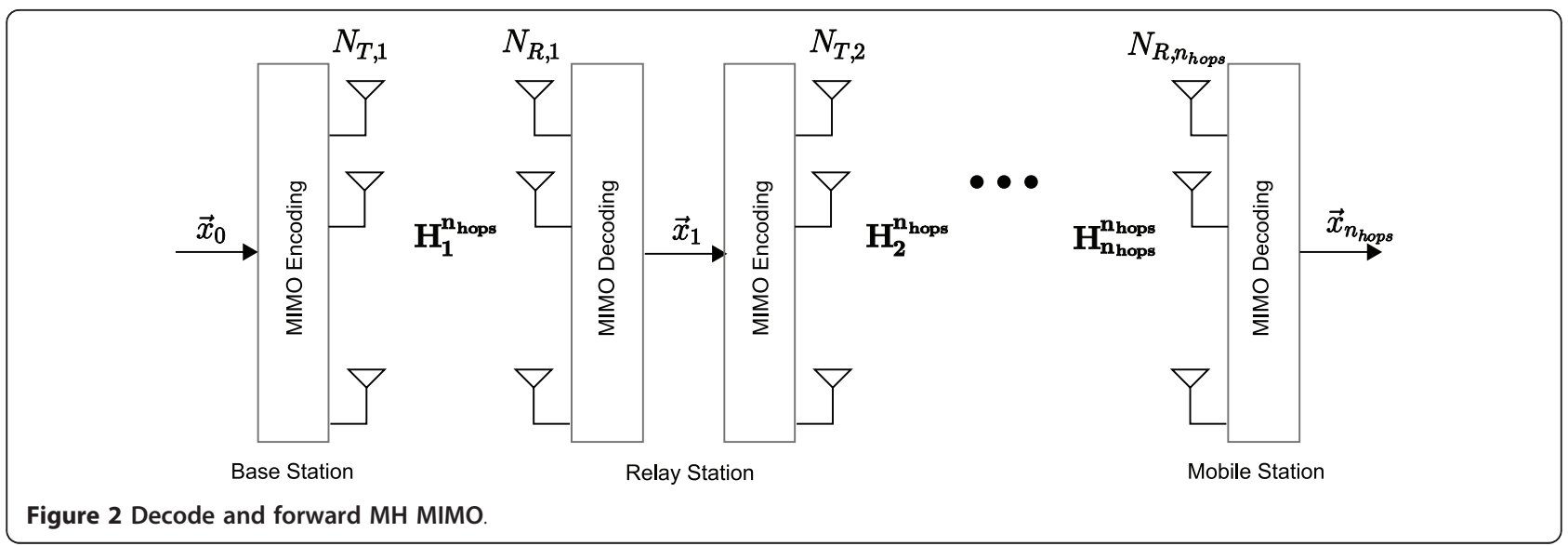

is optionally added in (7) to model random shadowing effects. $\psi_{\mathrm{dB}}$ has zero mean, and its standard deviation, $\sigma_{\psi \mathrm{dB}}$, is typically $10 \mathrm{~dB}$ in an urban NLOS microcell, and $4 \mathrm{~dB}$ in an urban LOS microcell [22].

Similarly, the Rice factor, $K_{r}(x)$, is modeled as a function of distance $[22,33]$

$$
K_{r}(x)= \begin{cases}0 & b<x<5,000 \mathrm{~m} \\ 10^{1.3-0.003 x} & 20 \mathrm{~m}<x<b\end{cases}
$$

From (7) and (8), we can see that the channel matrix elements are modeled as Rayleigh random variables when $b<x<5,000 \mathrm{~m}$ and Ricean (with $K_{r}>0$ ) when $20 \mathrm{~m}<x<b$. This is a general and simple method of modeling the channel for the purposes of studying the interaction of MH relaying and MIMO in this paper. The precise RF propagation characteristics of a system will depend on the specific location, and a more accurate RF propagation simulation would be required. However, we believe that this simple model will enable sufficient insight into the system behaviour.

Capacity (normalized by bandwidth so that it is expressed in $\mathrm{b} / \mathrm{s} / \mathrm{Hz}), R_{k}^{n_{\text {hops }}}$ of the $k$ th hop is a function $R(\cdot)$ (using (2), (3) or (5) as appropriate) of the channel realization for the hop:

$$
R_{k}^{n_{\text {hops }}}=R\left(\mathbf{H}_{k}^{n_{\text {hops }}}\right), k=1,2, \ldots, n_{\text {hops }}
$$

When calculating the SINRs for the hops, interference from all other transmitting stations is included, at levels determined by their transmit powers, distances from the receiver, and antenna gains (see [25-27] for the detailed parameters). In $\mathrm{MH}$ relaying, interfering stations are usually far enough away that their signals experience the higher NLOS path loss.

The primary system parameters used are summarized in Table 1.

We have simulated cases, in which each hop uses $\left(N_{T}\right.$, $\left.{ }_{k} \times N_{R, k}\right)=(1 \times 1)$ (single antenna), $(2 \times 2),(3 \times 3),(4$ $\times 4),(5 \times 5)$, and $(6 \times 6)$ MIMO. In practice, the BS can have a large antenna array, RSs must have a smaller array since they must be smaller and inexpensive, and MSs (laptop computers or mobile computing devices) are very limited in size. So we simulated a more realistic case (called the Mixed case in the figures), as described in Table 2. This creates hops with $\left(N_{T, k} \times N_{R, k}\right)=(4 \times$ $3),(3 \times 3)$, and $(3 \times 2)$ on the downlink BS-RS, RS-RS, and RS-MS hops, respectively. The uplink will have $\left(N_{T}\right.$, $\left.{ }_{k} \times N_{R, k}\right)=(2 \times 3),(3 \times 3)$, and $(3 \times 4)$ on the MS-RS, RS-RS, and RS-BS hops, respectively.

\section{3) Two-dimensional multihop cellular system}

The one-dimensional model can be extended to two dimensions in order to simulate a two-dimensional cellular layout. A cellular system is composed of numerous cells covering a large area. These cells are normally approximated as tessellating equal-size hexagons in most greenfield scenarios, or as equal-size squares in a downtown urban street scenario (Manhattan). A base station (BS) is deployed in the center of each cell and serves numerous mobile stations (MSs) in that cell. All frequency channels are reused in each cell (universal

\section{Table 1 Model parameters}

\begin{tabular}{ll}
\hline Carrier frequency, $f_{C}$ & $5.8 \mathrm{GHz}$ \\
Channel bandwidth, $W$ & $10 \mathrm{MHz}$ \\
Receiver noise figure, $F$ & $8 \mathrm{~dB}$ \\
Maximum transmit power, $P_{T X}$ & $30 \mathrm{dBm}$ \\
Omni antenna gain, $G_{T X}, G_{R X}$ & $9 \mathrm{dBi}$ \\
Directional antenna gain, $G_{T X}, G_{R X}$ & $17.5 \mathrm{dBi}$ \\
Directional antenna front-back ratio, $G_{F B}$ & $25 \mathrm{~dB}$ \\
Link margin, $M$ & $5 \mathrm{~dB}$ \\
PHY mode & 802.16 OFDM256 \\
BS antenna height & $32 \mathrm{~m}$ \\
Subcell RS antenna height & $10 \mathrm{~m}$ \\
MS antenna height & $1.5 \mathrm{~m}$ \\
Building height & $12 \mathrm{~m}$ \\
\hline
\end{tabular}


Table 2 Mixed MH MIMO case

\begin{tabular}{ccc}
\hline & $\boldsymbol{N}_{\boldsymbol{T}}$ & $\boldsymbol{N}_{\boldsymbol{R}}$ \\
\hline BS & 4 & 4 \\
RS & 3 & 3 \\
MS & 2 & 2 \\
\hline
\end{tabular}

frequency reuse), which results in high co-channel interference from one cell to another. This is mitigated by using $\mathrm{MH}$ relaying as shown in [27]. In an $\mathrm{MH}$ relaying cellular system, numerous relay stations (RSs) are deployed throughout the cell, which subdivides the cell into numerous subcells. A cellular system is best served using regularly-placed fixed relays (infrastructure-based relaying). Figure 3 shows examples of hexagonal and Manhattan topologies for the four-hop relaying case. In cellular systems, data connections occur between each MS and the BS, which creates bottlenecks on links toward the BS.

MSs will be served by the closest RS or BS, handing off as necessary to a closer station as the MS moves. As a result, some MSs will obtain service directly from the BS (one hop), some MSs will be served by RSs via two or more hops depending on their locations. MSs at the cell edge will be served via the maximum number of hops in the cell. Wireless transport links exist between the BS and its closest RSs, and between adjacent RSs, and access links exist between a MS and its serving RS or BS. We consider only decode and forward relaying, in which the data stream is decoded and re-encoded at RSs before transmitting on the next hop. All relay stations are wireless and may not transmit and receive simultaneously (half-duplex). We can calculate the signal to interference and noise ratio (SINR) at each station's receiver and then find the rate attainable on each hop using a process similar to that described for the onedimensional network.

\section{B MAC layer}

The previous section described the calculation of $\mathrm{PHY}$ layer capacities of each hop. But the key measure of performance of MH MIMO in a cellular system is the overall achievable network capacity, $R_{\text {Net }}$. The MAC layer coordinates transmissions as the data propagates from BS through RSs to the destination MSs, and so we must now consider network-wide scheduling of these transmissions in order to determine network capacity.

As a first step, we consider non-spatial reuse scheduling, in which only one station in the entire macrocell is allowed to transmit in a channel at a particular time. This is not an efficient use of bandwidth, so we also consider spatial reuse in which simultaneous transmissions occur in the macrocell. In order to avoid inter-station interference and to ensure that a station is guaranteed not to be transmitting at the same time it is receiving (Lane-man's half-duplex constraint [34]), stations close to (one hop away from) a transmitting station must remain silent.

\section{1) One-dimensional multihop relaying}

It can be easily shown that for a linear $\mathrm{MH}$ system as shown in Figure 1, the non-spatial reuse network capacity is

$$
R_{\mathrm{Net}, \mathrm{NoSR}}=\left(\sum_{k=1}^{n_{\mathrm{hops}}} \frac{1}{R_{k}^{n_{\mathrm{hops}}}}\right)^{-1}
$$

and the spatial reuse network capacity is

$$
R_{\text {Net, SR }}=\left[\max \left(\frac{1}{R_{1}^{n_{\text {hos }}}}, \frac{1}{R_{3}^{n_{\text {hops }}}}, \ldots, \frac{1}{R_{p}^{n_{\text {pos }}}}\right)+\max \left(\frac{1}{R_{2}^{n_{\text {hops }}}}, \frac{1}{R_{4}^{n_{\text {hops }}}}, \ldots, \frac{1}{R_{q}^{n_{\text {hops }}}}\right)\right]^{-1}
$$

where $p \leq n_{\text {hops }}$ is an odd integer and $q \leq n_{\text {hops }}$ is an even integer.

\section{2) Two-dimensional multihop cellular system}

$R_{\text {Net }}$ in a two-dimensional system can be calculated knowing the data rates achievable on each of the links, and by considering the spatial reuse schedule imposed by the medium access control layer (MAC). With spatial reuse, data transmission can occur simultaneously in numerous subcells within the cell. The details of spatial reuse as applied to $\mathrm{MH}$ relaying have been presented in [27]. Expressions for $R_{\mathrm{Net}}$ have been derived for up to four-hop hexagonal and Manhattan cellular topologies. These expressions are used to obtain the results presented here.

\section{Results}

\section{A Single MIMO hop}

Here, we look at the performance of a single Ricean MIMO hop. As discussed earlier, the addition of relays shortens the hop distances, which reduces path loss and scattering (i.e., increases the Ricean factor $K_{r}$ ). It is useful to look at this effect on a single hop link before studying the full network. Figure 4 shows the average mutual information for a $(4 \times 4)$ MIMO link with full rank $\mathbf{H}_{\mathrm{NLOS}}$ and rank $1 \mathbf{H}_{\mathrm{LOS}}$ calculated from (5) [29].

Cellular systems generally operate at a fairly low SINR. It is easy to see from this figure that the rate advantage due to MIMO is relatively low at low SINR. We can increase the SINR on each hop by adding relays, but this may increase $K_{r}$, which reduces the MIMO capacity gain, until at $K_{r}=\infty$, there remains only $6 \mathrm{~dB}$ array gain due to multiple receive antennas. From (8) we find that $K_{r}$ is still about 10 at a fairly short distance of $100 \mathrm{~m}$, and so MIMO gain, although reduced at this distance, is not completely lost.

Figure 5 shows the dependence of capacity on the Rice factor and antenna configuration. More antennas do 


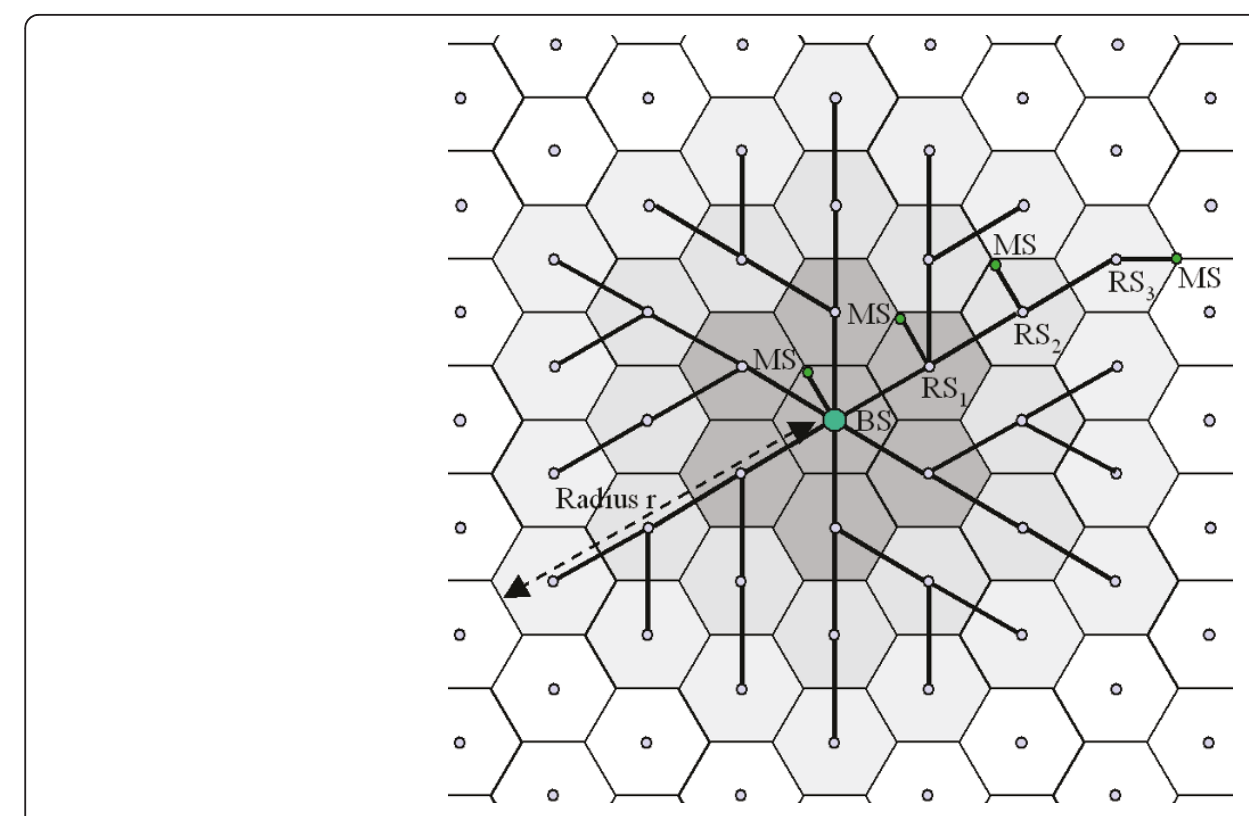

(a) Four hop hexagonal: 37 shaded subcells comprise one cell.

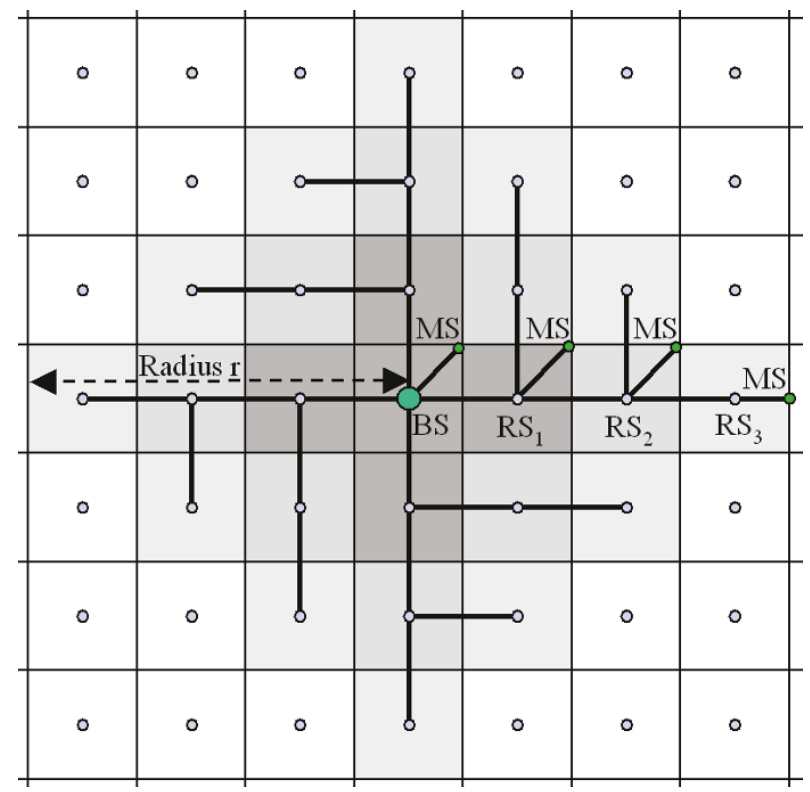

(b) Four hop Manhattan:25 shaded subcells com-

prise one cell.

Figure 3 Multihop relay cellular topologies. a Four hop hexagonal: 37 shaded subcells comprise one cell. b Four hop Manhattan: 25 shaded subcells comprise one cell.

provide higher capacities, but the loss in capacity with increasing $K_{r}$ is greater.

Figure 6 shows the dependence of capacity on the Rice factor and SINR. The plots show that the capacity can drop off quite drastically with $K_{r}$ at a fixed SINR, especially with a large number of antennas. Rice factor in cellular systems typically ranges from 3 to 20 , which is in the range of steep reduction of capacity. 


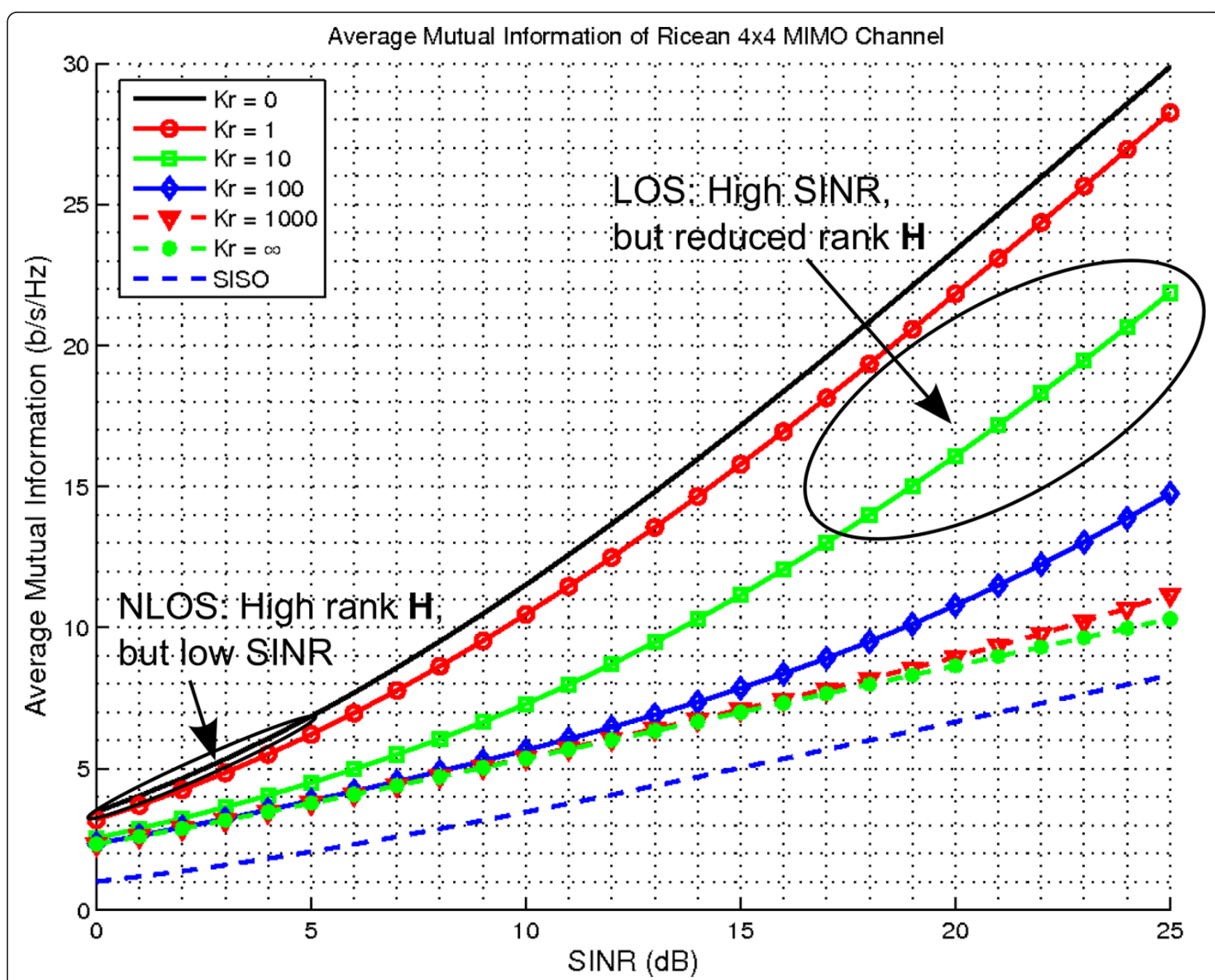

Figure 4 Upper bound on the average mutual information for $(4 \times 4)$ Ricean MIMO hop, with full rank $\mathrm{H}_{\mathrm{NLOS}}$ and rank $1 \mathrm{H}_{\text {LOS, }}$ and a comparison to SISO.

The previous results show the effects of $K_{r}$ and SINR with one of them fixed while we vary the other. However, Rice factor and path loss change simultaneously with distance in a real propagation environment, since a rich scattering environment (which is good for MIMO) also becomes depleted with decreasing LOS path loss. In the following figures, we examine the effects of $K_{r}$ and SINR jointly using the $K_{r}(x)$ and $\rho(x)$ models given by (7) and (8). Figure 7a shows how $K_{r}$ and path loss vary with distance, using a distance breakpoint of $300 \mathrm{~m}$. Figure 7b shows the resulting hop capacity. It is clear that the loss in MIMO gain is small compared to the gain due to increased SINR.

\section{B One-dimensional multihop relaying}

In this section, we look at how MIMO and MH relaying operate together in a one-dimensional linear system with co-channel interference. Numerous cases have been simulated using the system model as described. We include here a sample of simulation results, for up to eight hops, and up to $(6 \times 6)$ MIMO. Figures 8 and 9 show some sample results for a cell radius of $1,500 \mathrm{~m}$, equally spaced relays and a distance breakpoint of $300 \mathrm{~m}$. For fewer than six hops, all hops are NLOS and so the path loss of each hop is high. All hop paths are uncorrelated Rayleigh channels, which should provide a good environment for capacity gain due to MIMO spatial multiplexing. However, the hops suffer from low SINR due to high path loss and cochannel interference. Since spatial multiplexing works best at high SINR, MIMO capacity gain is minimal. With the addition of another relay (a sixth hop), all hops become LOS and the path loss of each hop becomes drastically reduced. As a result, the hop SINRs increase and the network capacity increases greatly. Although SINR is much higher, spatial multiplexing and diversity gains suffer due to the largely correlated propagation environment. However, MIMO does assist in MH LOS situations because 


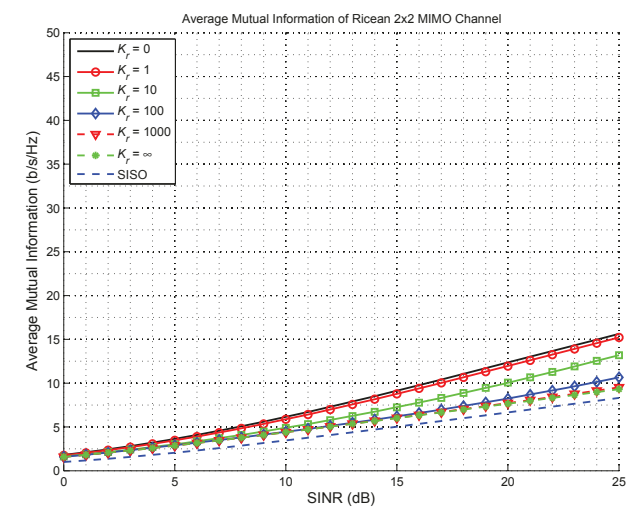

(a) $\left(N_{R} \times N_{T}\right)=(2 \times 2)$.

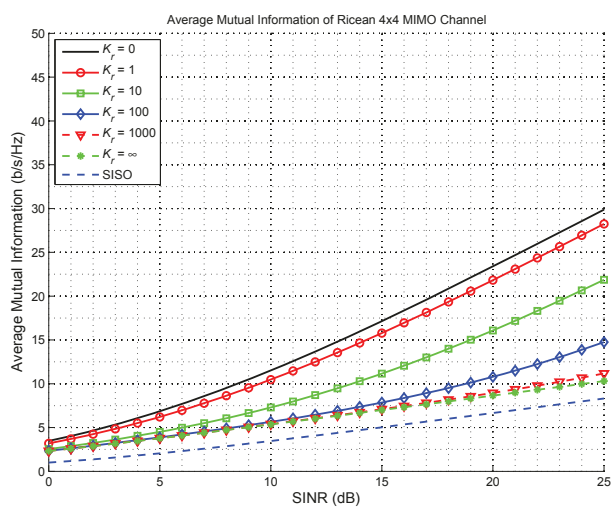

(c) $\left(N_{R} \times N_{T}\right)=(4 \times 4)$.

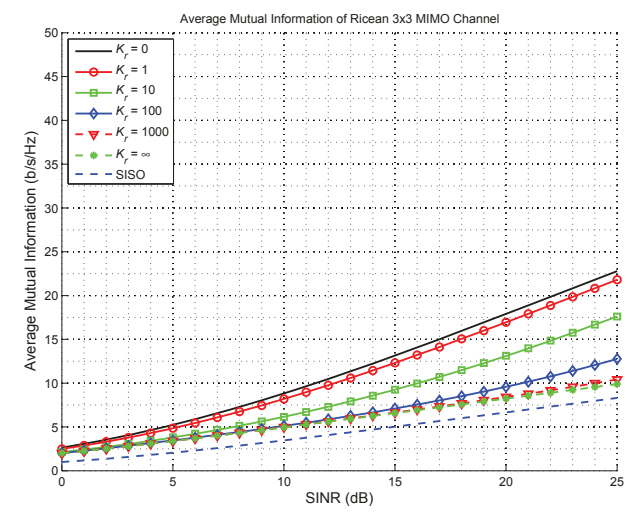

(b) $\left(N_{R} \times N_{T}\right)=(3 \times 3)$.

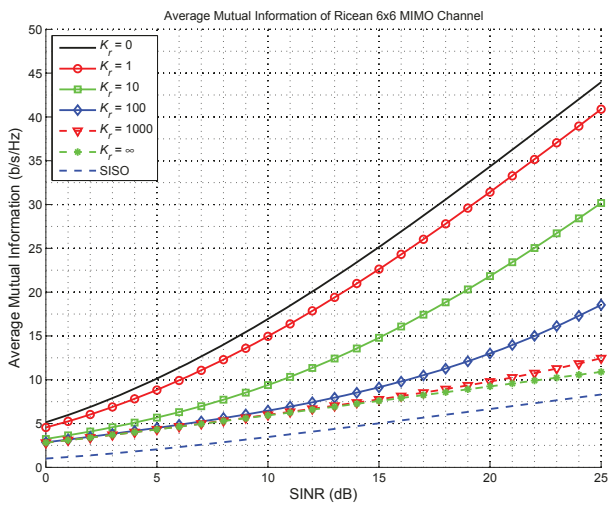

(d) $\left(N_{R} \times N_{T}\right)=(6 \times 6)$.

Figure 5 Upper bound on the average mutual information for a Ricean MIMO hop, with full rank $\mathbf{H}_{\text {NLOs }}$ and rank $\mathbf{1} \mathbf{H}_{\mathrm{LOS}}$ a $\left(N_{R} \times N_{T}\right)=$ $(2 \times 2)$. $\mathbf{b}\left(N_{R} \times N_{T}\right)=(3 \times 3)$. $\mathbf{c}\left(N_{R} \times N_{T}\right)=(4 \times 4)$. $\mathbf{d}\left(N_{R} \times N_{T}\right)=(6 \times 6)$.

there remains some scattering component, and there exist receive array gain and interference control afforded by conventional transmit beamforming.

Figure 8 clearly shows the importance of spatial reuse in $\mathrm{MH}$ relaying. When there are more than two hops, channels (time or frequency slots) can be reused at stations that are adequately separated in space, which provides great increases in network-wide spectral efficiency despite the introduction of interference between subcells. Without spatial reuse, interference is lower, but $\mathrm{MH}$ relaying is more wasteful of spectrum. As shown in Figure $8 \mathrm{a}$, no spatial reuse case, $R_{\mathrm{Net}}$, decreases beyond 6 hops since relaying is increasingly wasteful of resources. With fewer than 6 hops, the addition of relays is slightly beneficial since the increase in SINR afforded by shortening the hop distances increases the MIMO gain. In Figure $8 \mathrm{~b}$, with spatial reuse, $R_{\text {Net }}$ continuously increases with the number of hops. With more relays, there is more opportunity for channel reuse in distant parts of the cell.
Cumulative distribution functions of MH MIMO network capacity for some cases are shown in Figure 9. The figure demonstrates the drastic capacity increase that $\mathrm{MH}$ relaying can achieve by avoiding NLOS propagation and enabling spatial reuse, and the gradual increase in capacity afforded by MIMO.

Figures 8 and 9 show the results using a rank one $\mathbf{H}_{\mathrm{LOS}, k}^{n_{\text {hops }}}$, while Figures 10 and 11 show the results for full rank $\mathbf{H}_{\mathrm{LOS}, k}^{n_{\text {hop }}}$. The results are similar, but obviously $R_{\mathrm{Net}}$ is higher when the LOS matrix is high rank (although this is not likely to occur in a real cellular system [29]).

\section{Two-dimensional multihop cellular system}

In this section, we extend the calculations to a cellular system with tesselated Manhattan and hexagonal cells with one to four hops using the results of [27].

Universal frequency reuse is used among the cells for all cases. We assume the use of omnidirectional (in the 


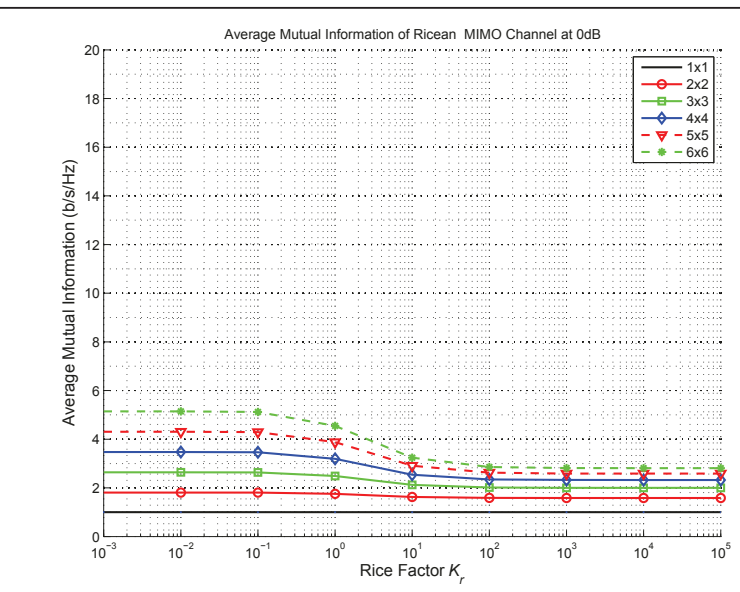

(a) $\operatorname{SINR} \rho=0 \mathrm{~dB}$.

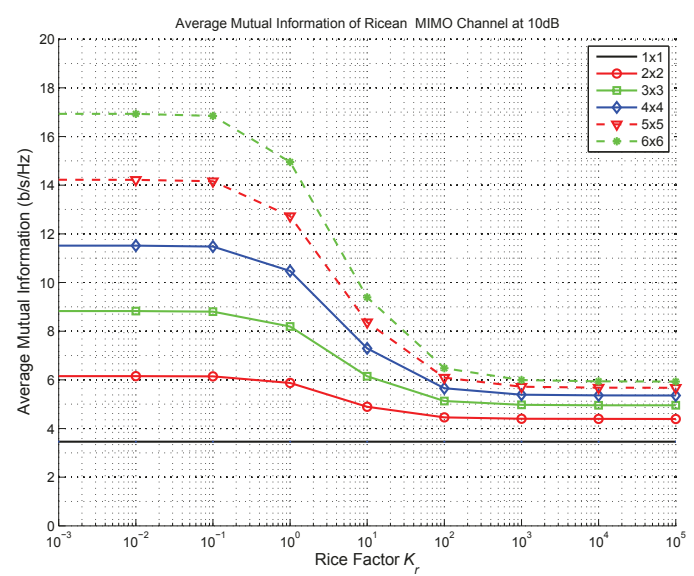

(c) $\operatorname{SINR} \rho=10 \mathrm{~dB}$.

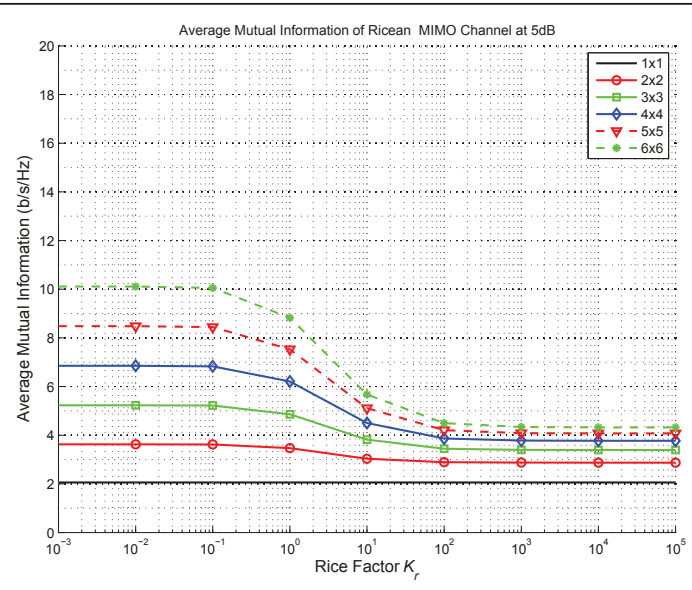

(b) $\operatorname{SINR} \rho=5 \mathrm{~dB}$.

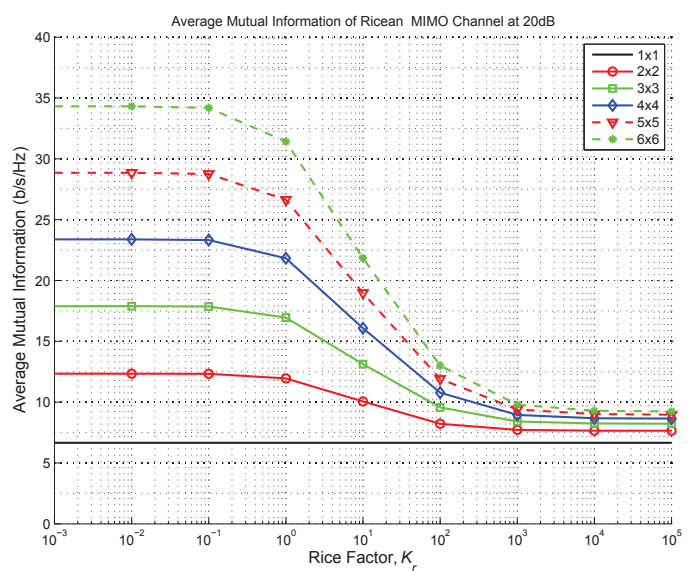

(d) $\operatorname{SINR} \rho=20 \mathrm{~dB}$.

Figure 6 Upper bound on the average mutual information for a Ricean MIMO hop, with full rank $\mathrm{H}_{\text {NLOS }}$ and rank $1 \mathrm{H}_{\text {LOS. }}$ a SINR $\rho=0$ dB. b SINR $\rho=5 \mathrm{~dB}$. c SINR $\rho=10 \mathrm{~dB}$. d SINR $\rho=20 \mathrm{~dB}$.

horizontal plane) antenna elements for the MIMO arrays since they provide the greatest spatial spread.

For a detailed example, we show calculations for a hexagonal topology with circumscribed cell radius of $500 \mathrm{~m}$. The hop distances for this case are given in Table 3. The resulting SINRs are given in Table 4.

It is useful to observe how distances, path losses, and SINRs change as relays are added to this system. The non-linear path loss model used, combined with the effect of scheduling transmissions among subcells within a cell, gives some non-linear and somewhat surprising results.

With no relays $(n=1)$, an MS at the cell edge is 500 $m$ from the BS, which gives a NLOS channel according to the path loss model (7). In this case, reception at the MS suffers from high co-channel interference from adjacent cells and a very poor SINR since we are considering universal frequency reuse among cells. The twohop $(n=2)$ hexagonal case has six RSs around the BS that gives two hops between the BS and any MS at the cell edge. The first hop, between the BS and any RS, is about $333 \mathrm{~m}$ and therefore is Rayleigh/NLOS according to the dual slope model. The second hop, between any RS and a cell-edge MS, is about $167 \mathrm{~m}$ and Ricean/LOS. The first-hop link suffers from high path loss, and experiences high co-channel interference from numerous RSs in other cells. In fact, there are three interfering RSs in other cells that are the same distance away as the BS. The interference is particularly bad from those RSs since the scheduling of RS transmissions in the other cells is not coordinated with the BS and RSs in the studied cell. Interference from within the studied cell is 


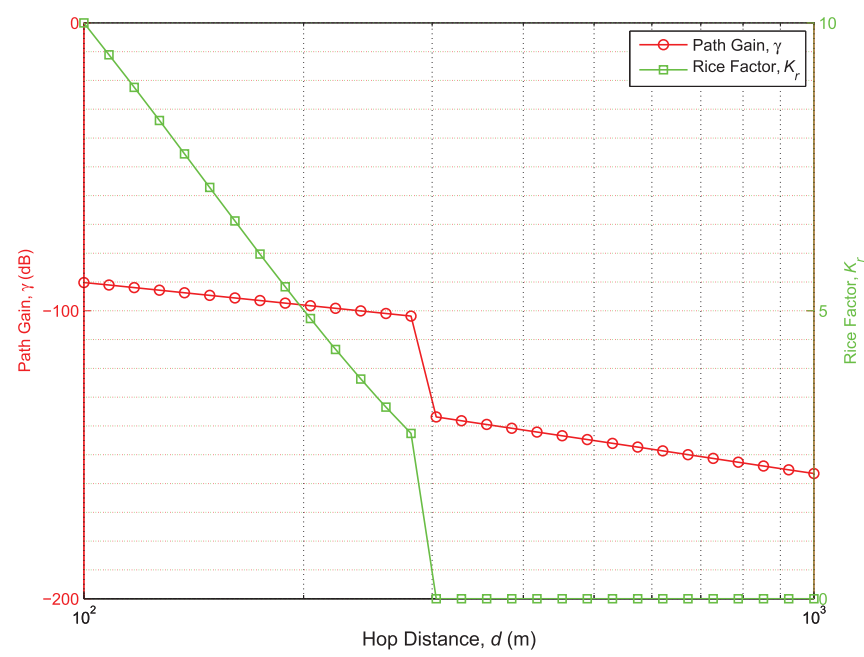

(a) Dependenceof path loss and Rice factor on hop distance.

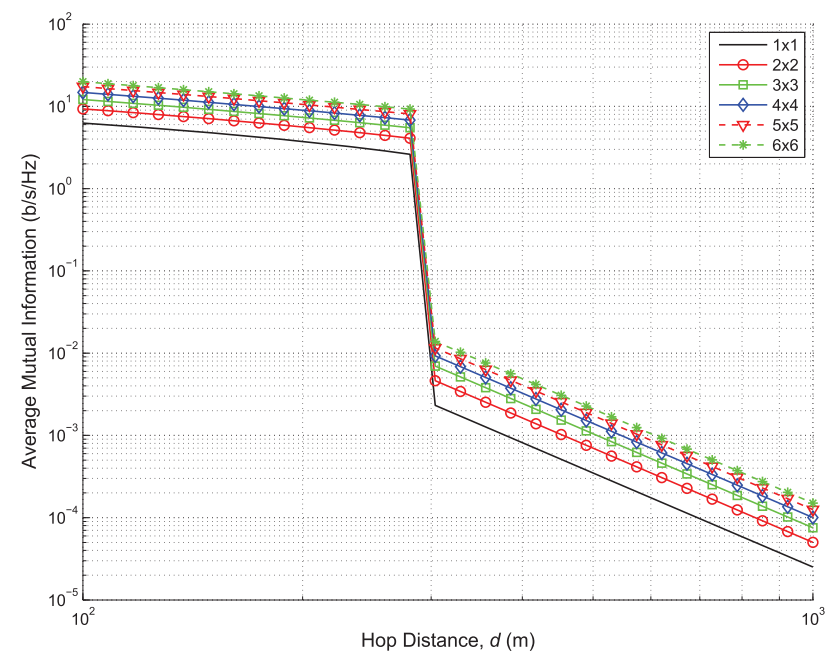

(b) Dependenceof average mutual information for Ricean MIMO channels on hop distance, with full rank $\mathrm{H}_{\mathrm{NL}}$ os and rank $1 \mathrm{H}_{\text {LO }} \mathrm{s}$ and fixed transmit power, $P_{T X}=0 \mathrm{dBm}$.

Figure 7 Effect of hop distance, using dual slope model. a Dependence of path loss and Rice factor on hop distance. $\mathbf{b}$ Dependence of average mutual information for Ricean MIMO channels on hop distance, with full rank $\mathbf{H}_{\mathrm{NLOS}}$ and rank $1 \mathbf{H}_{\llcorner\mathrm{LOS}}$ and fixed transmit power, $P_{T X}=0$ $\mathrm{dBm}$. 


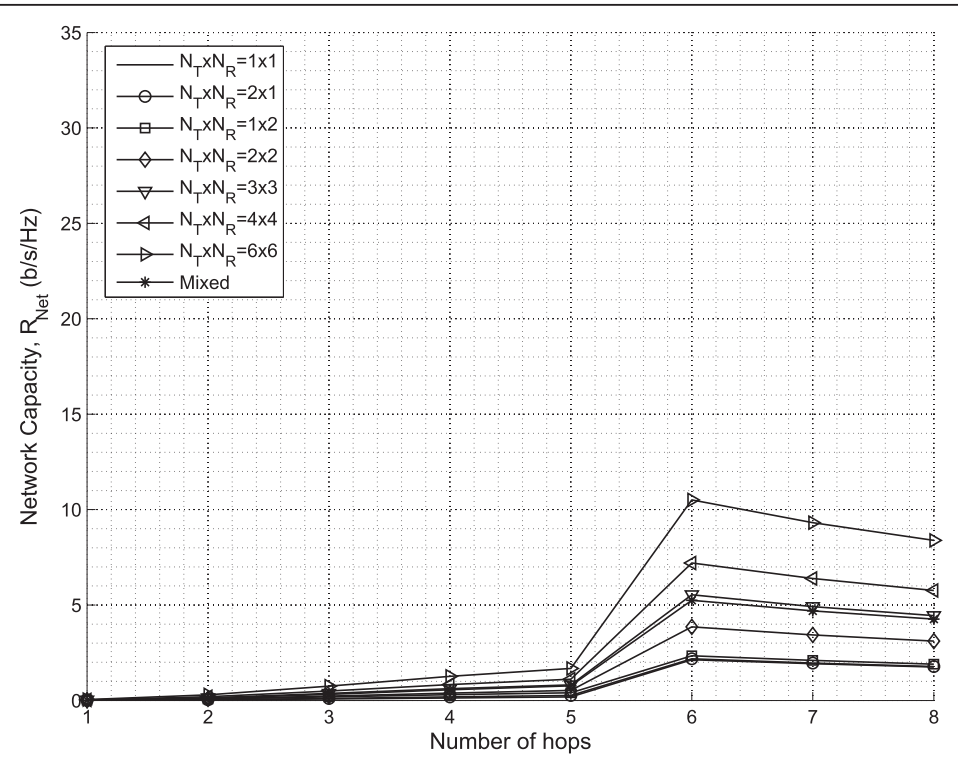

(a) Multihop MIMO - no spatial reuse.

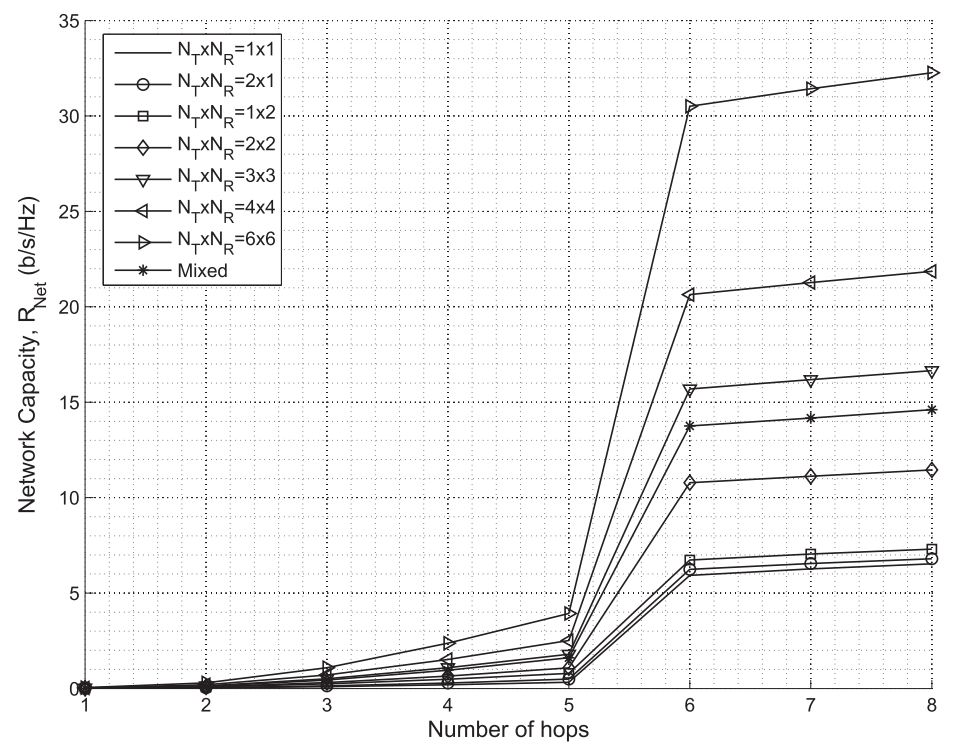

(b) Multihop MIMO with spatial reuse.

Figure 8 Multihop MIMO network capacities $\left(\boldsymbol{R}_{\mathrm{Net}}\right)$-with rank one LOS channel matrices. a Multihop MIMO-no spatial reuse. b Multihop MIMO with spatial reuse. 


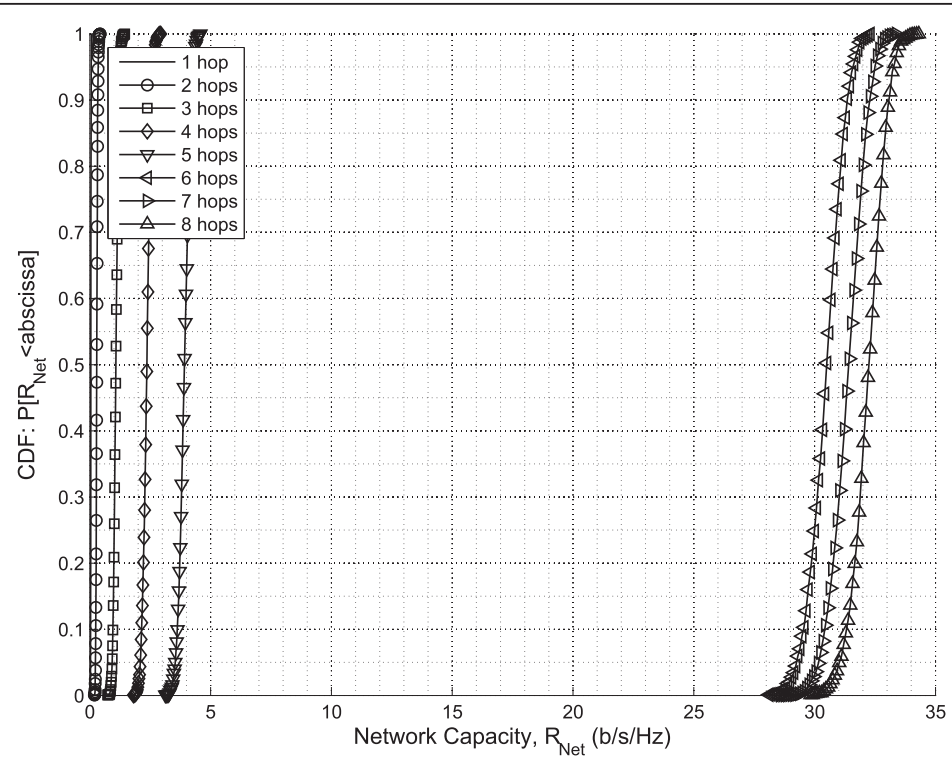

(a) Multihop, $(6 \times 6)$ MIMO with spatial reuse.

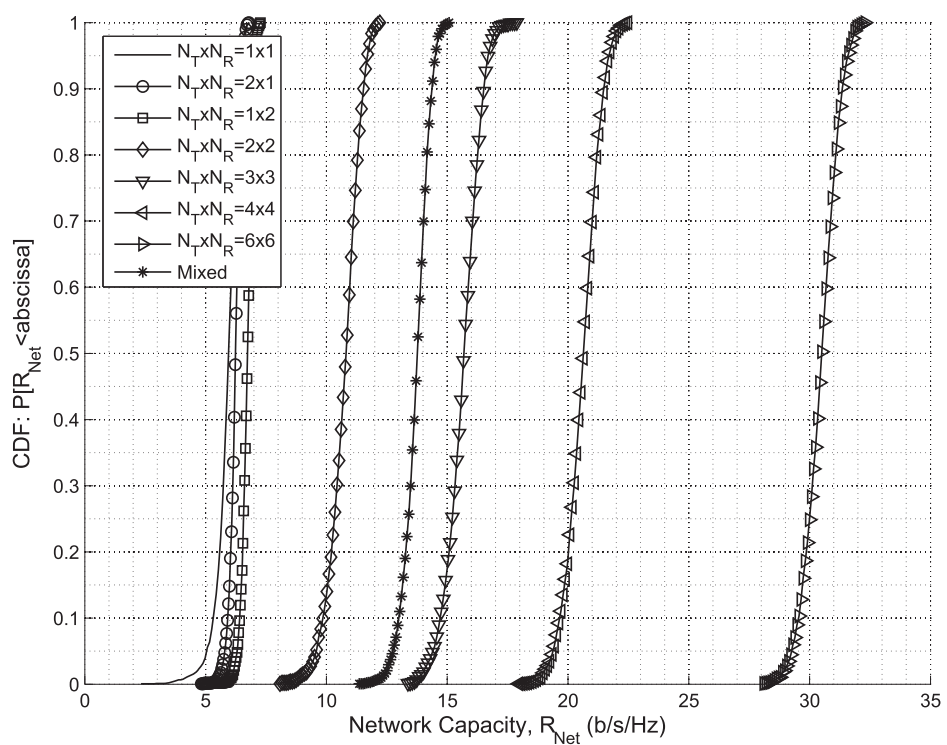

(b) Six hop MIMO with spatial reuse.

Figure 9 Cumulative distribution functions of MH MIMO network capacity $\left(R_{\mathrm{Net}}\right)$-with rank one LOS channel matrices. a Multihop, $(6 \times$ 6) MIMO with spatial reuse. b Six hop MIMO with spatial reuse. 


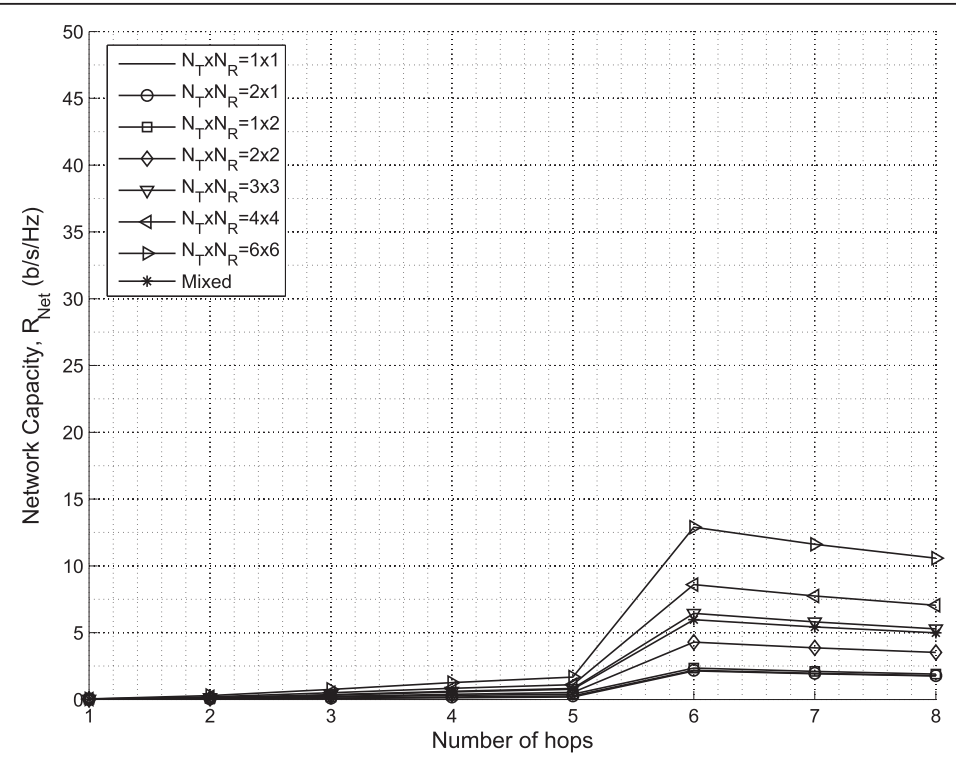

(a) Multihop MIMO - no spatial reuse.

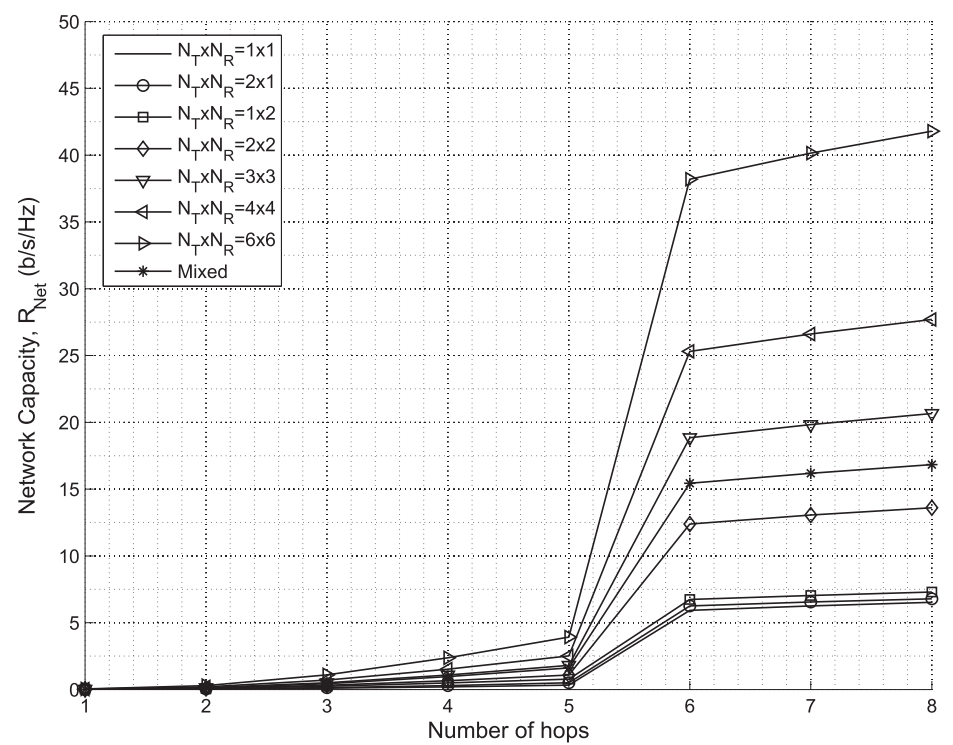

(b) Multihop MIMO with spatial reuse.

Figure 10 Multihop MIMO network capacities $\left(\boldsymbol{R}_{\text {Net }}\right)$-with full rank LOS channel matrices. a Multihop MIMO-no spatial reuse. b Multihop MIMO with spatial reuse. 


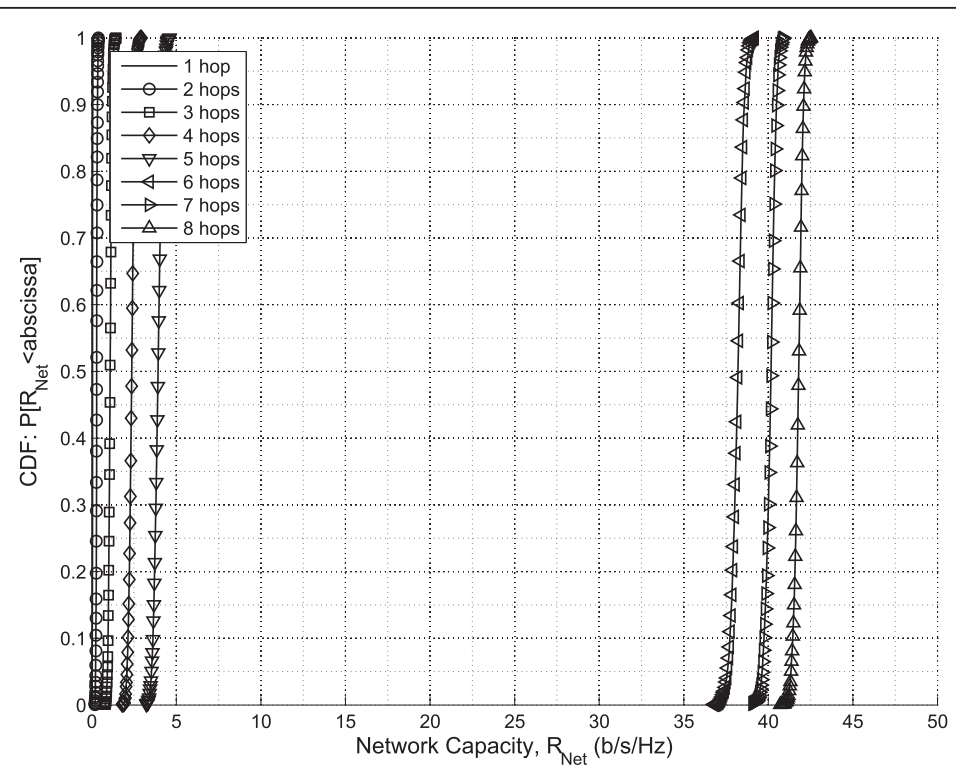

(a) Multihop, $(6 \times 6)$ MIMO with spatial reuse.

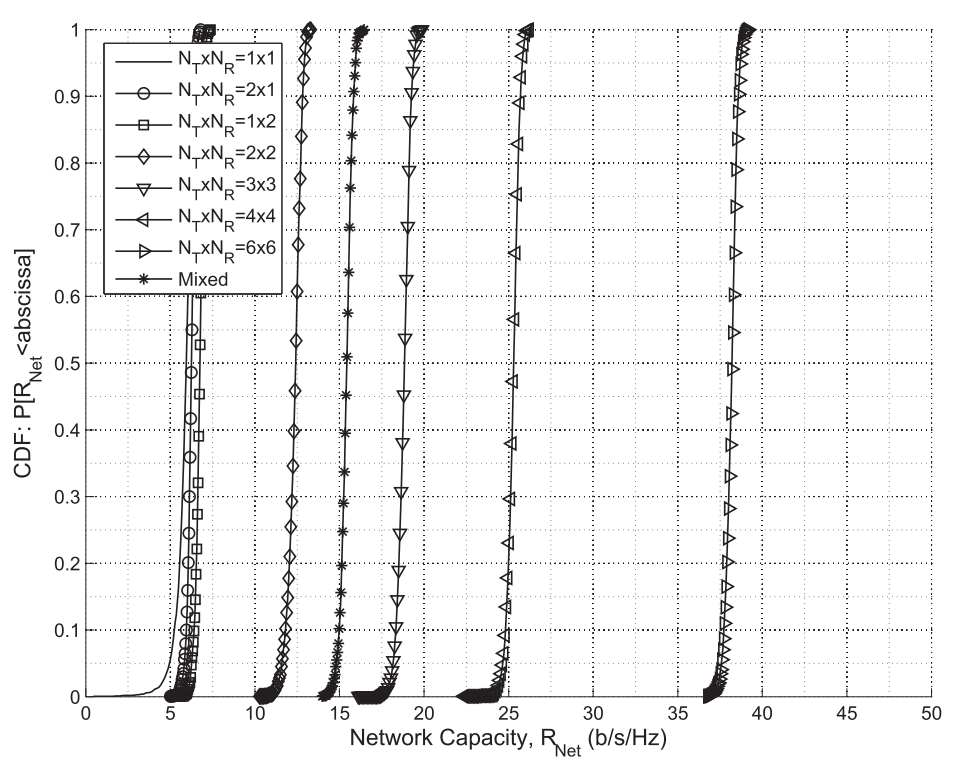

(b) Six hop MIMO with spatial reuse.

Figure 11 Multihop MIMO cumulative distribution functions $\left(\boldsymbol{R}_{\text {Net }}\right)$-with full rank LOS channel matrices. a Multihop, $(6 \times 6)$ MIMO with spatial reuse. b Six hop MIMO with spatial reuse. 
Table 3 Hop distances: $\mathbf{5 0 0} \mathrm{m}$ radius hexagonal cell

\begin{tabular}{ccccc}
\hline & \multicolumn{4}{c}{ Distance per hop (m) and path type (NLOS/LOS) } \\
\cline { 2 - 5 } $\boldsymbol{n}$ & $\boldsymbol{r}_{\mathbf{1}}$ & $\boldsymbol{r}_{\mathbf{2}}$ & $\boldsymbol{r}_{\mathbf{3}}$ & $\boldsymbol{r}_{\mathbf{4}}$ \\
\hline 1 & 500-NLOS & - & - & - \\
2 & $333-N L O S$ & $167-$-LOS & - & - \\
3 & 200-LOS & 200-LOS & 100-LOS & - \\
4 & 143-LOS & 143-LOS & 143-LOS & 71-LOS \\
\hline
\end{tabular}

eliminated by scheduling. The second hop has a much better SINR since that link enjoys a much reduced path loss due to LOS, yet interfering signals are a greater distance away and experience higher losses due to NLOS.

Adding 12 more RSs creates a three-hop hexagonal system. All three hops to an MS at the cell edge are LOS channels but the interfering channels are still NLOS. Also, RSs within the studied cell can be scheduled to minimize co-channel interference. Interfering RSs in other cells, uncoordinated with transmissions in the study cell, are now a much greater distance away and so have much less impact than in the two-hop case. The resulting improvement in SINR on the links is dramatic.

The next step, creating a four-hop hexagonal system, shortens the hops a little more. However, the incremental improvement over three-hop is less dramatic since LOS links were already obtained by the three-hop system. Notice that the SINR has improved on the first hop fairly significantly since the inner RSs become more insulated from the interfering transmissions from other cells. The last hop does not improve much in SINR because it is still quite near interfering subcells in the adjacent cells.

With the SINRs calculated above, we can now calculate the rates on each hop, and the aggregate network rate, $R_{\text {Net }}$, with spatial reuse. Single antenna, $(3 \times 3)$ MIMO, and mixed MIMO cases are shown in Tables 5 , 6 , and 7 , respectively.

Figure 12 compares the aggregate bit rates achievable by numerous MIMO configurations versus $n_{\text {hops }}$, for Manhattan and hexagonal topologies with $500 \mathrm{~m}$ radius cells. Figure 13 shows the results for 1,000 m radius cells.

\section{Discussion}

Results of this work show that there is a fundamental capacity tradeoff when using MIMO and MH relaying

Table 4 SINR: $500 \mathrm{~m}$ radius hexagonal cell

\begin{tabular}{ccccc}
\hline & \multicolumn{4}{c}{ SINR per hop (dB) } \\
\cline { 2 - 5 } $\boldsymbol{n}$ & Hop 1 & Hop 2 & Hop 3 & Hop 4 \\
\hline 1 & -5.4 & - & - & - \\
2 & -12.9 & 19.4 & - & - \\
3 & 28.9 & 19.8 & 17.4 & - \\
4 & 32.5 & 27.9 & 18.4 & 16.1 \\
\hline
\end{tabular}

Table 5 Rates: $500 \mathrm{~m}$ radius hexagonal cell, single antenna

\begin{tabular}{|c|c|c|c|c|c|}
\hline \multirow[b]{2}{*}{$n_{\text {hops }}$} & \multicolumn{4}{|c|}{$R$ per hop $(\mathrm{b} / \mathrm{s} / \mathrm{Hz})$} & \multirow[t]{2}{*}{$R_{\text {net }}(\mathrm{b} / \mathrm{s} / \mathrm{Hz})$} \\
\hline & Hop 1 & Hop 2 & Hop 3 & Hop 4 & \\
\hline 1 & 0.30 & - & - & - & 0.30 \\
\hline 2 & 0.058 & 6.2 & - & - & 0.067 \\
\hline 3 & 9.3 & 6.3 & 5.5 & - & 5.2 \\
\hline 4 & 10.5 & 8.9 & 5.8 & 5.1 & 7.7 \\
\hline
\end{tabular}

jointly. This may seem obvious, since the two techniques actually work using conflicting assumptions: MIMO works by exploiting the randomly scattering channel, while $\mathrm{MH}$ relaying attempts to mitigate that random behaviour. A key effect is the loss of MIMO's diversity and spatial multiplexing gains as relaying is introduced. This is apparent from (2) since, with $r_{\mathrm{LOS}}=$ 1 , the rank of $\mathbf{H}$ decreases and MIMO capacity gain is lost as the Rice factor, $K_{r}$, increases. However, multiple antennas provide advantages due to receive array gain, and due to minimization of co-channel interference with conventional transmit beamforming methods. Also, the use of $\mathrm{MH}$ relaying shortens the hop distances, which increases the SINR. So although scattering is reduced, SINR is increased. Increasing the SINR provides higher spatial multiplexing gain, but reducing scattering reduces spatial multiplexing gain. To put this another way, MIMO's spatial multiplexing and diversity gains are achieved at the expense of SINR: the uncorrelated signal that is key to MIMO gains occurs because the signal experiences rich scattering associated with high path loss.

One might expect that $\mathrm{MH}$ relaying should work best since it addresses the real root of the problem-a weak

Table 6 Rates: $500 \mathrm{~m}$ radius hexagonal cell, $(3 \times 3)$ MIMO on each hop

\begin{tabular}{|c|c|c|c|c|c|}
\hline \multirow[b]{2}{*}{$n_{\text {hops }}$} & \multicolumn{4}{|c|}{$R$ per hop $(\mathrm{b} / \mathrm{s} / \mathrm{Hz})$} & \multirow[t]{2}{*}{$R_{\text {net }}(\mathrm{b} / \mathrm{s} / \mathrm{Hz})$} \\
\hline & Hop 1 & Hop 2 & Hop 3 & Hop 4 & \\
\hline 1 & 1.0 & - & - & - & 1.0 \\
\hline 2 & 0.2 & 17.3 & - & - & 0.24 \\
\hline 3 & 23.0 & 14.3 & 11.0 & - & 12.1 \\
\hline 4 & 25.6 & 21.1 & 12.4 & 9.8 & 18.0 \\
\hline
\end{tabular}

Table 7 Rates: $\mathbf{5 0 0} \mathrm{m}$ radius hexagonal cell, mixed MIMO case

\begin{tabular}{|c|c|c|c|c|c|}
\hline \multirow[b]{2}{*}{$n_{\text {hops }}$} & \multicolumn{4}{|c|}{$R$ per hop $(\mathrm{b} / \mathrm{s} / \mathrm{Hz})$} & \multirow[t]{2}{*}{$R_{n e t}(\mathrm{~b} / \mathrm{s} / \mathrm{Hz})$} \\
\hline & Hop 1 & Hop 2 & Hop 3 & Hop 4 & \\
\hline 1 & 0.72 & - & - & - & 0.72 \\
\hline 2 & 0.21 & 12.4 & - & - & 0.25 \\
\hline 3 & 23.7 & 14.3 & 8.9 & - & 11.6 \\
\hline 4 & 26.3 & 21.1 & 12.4 & 7.9 & 17.5 \\
\hline
\end{tabular}




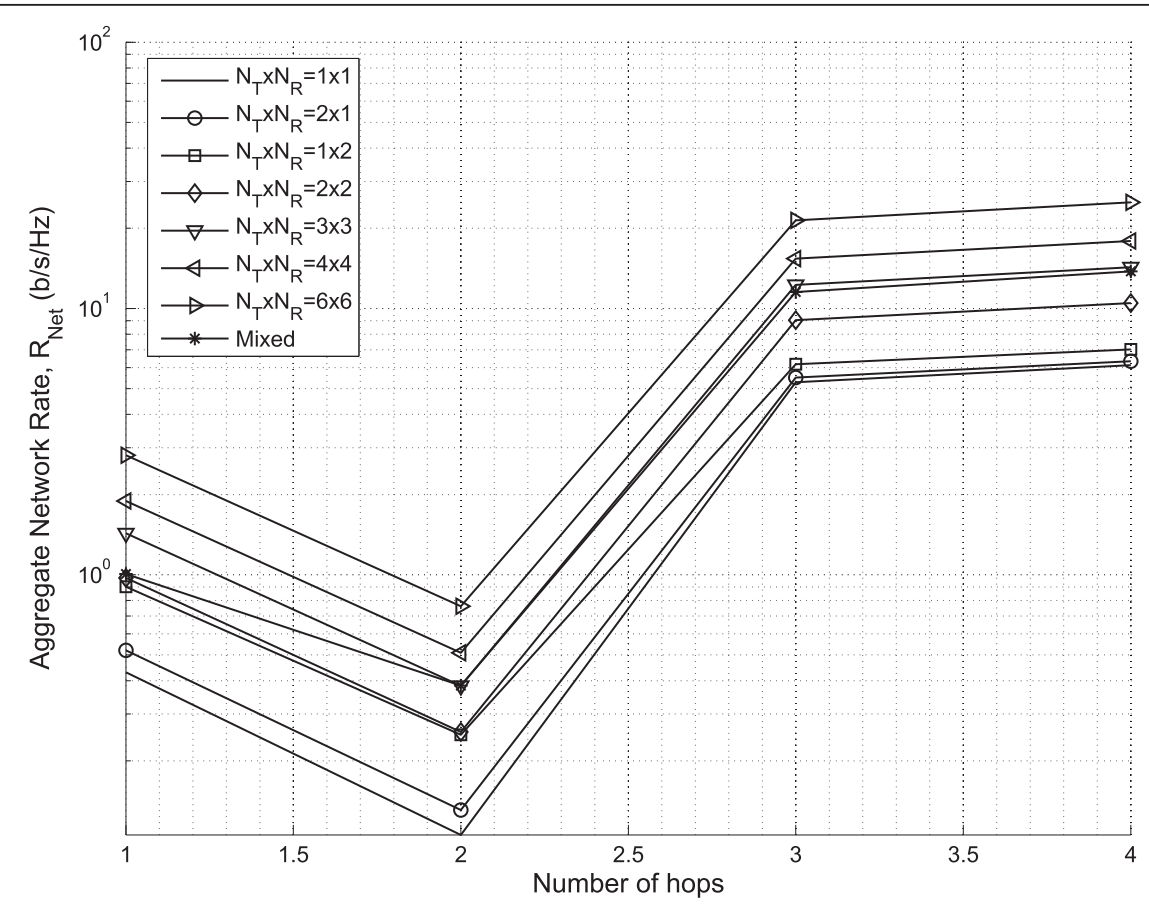

(a) ManhattanMH MIMO cell.

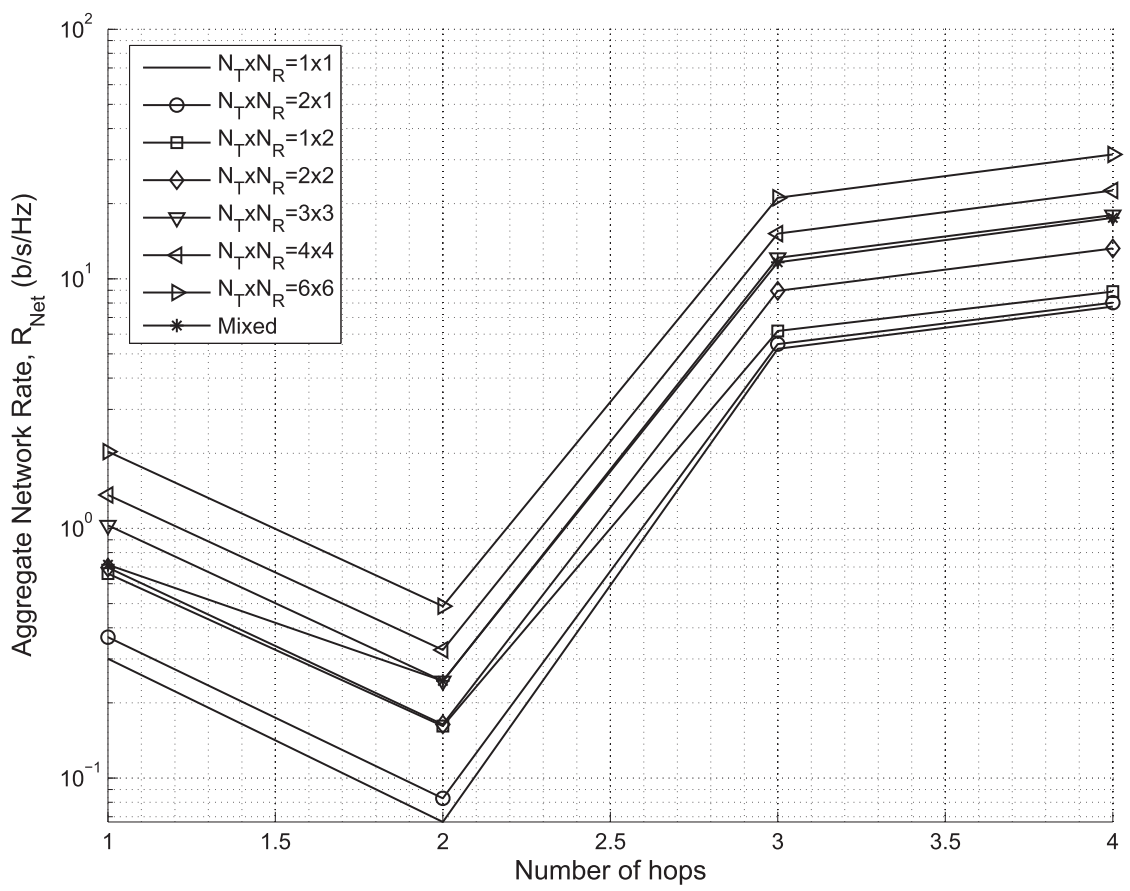

(b) Hexagonal MH MIMO cell.

Figure 12 Aggregate network rate for 500 m radius $\mathrm{MH}$ MIMO cells. a Manhattan MH MIMO cell. b Hexagonal MH MIMO cell. 


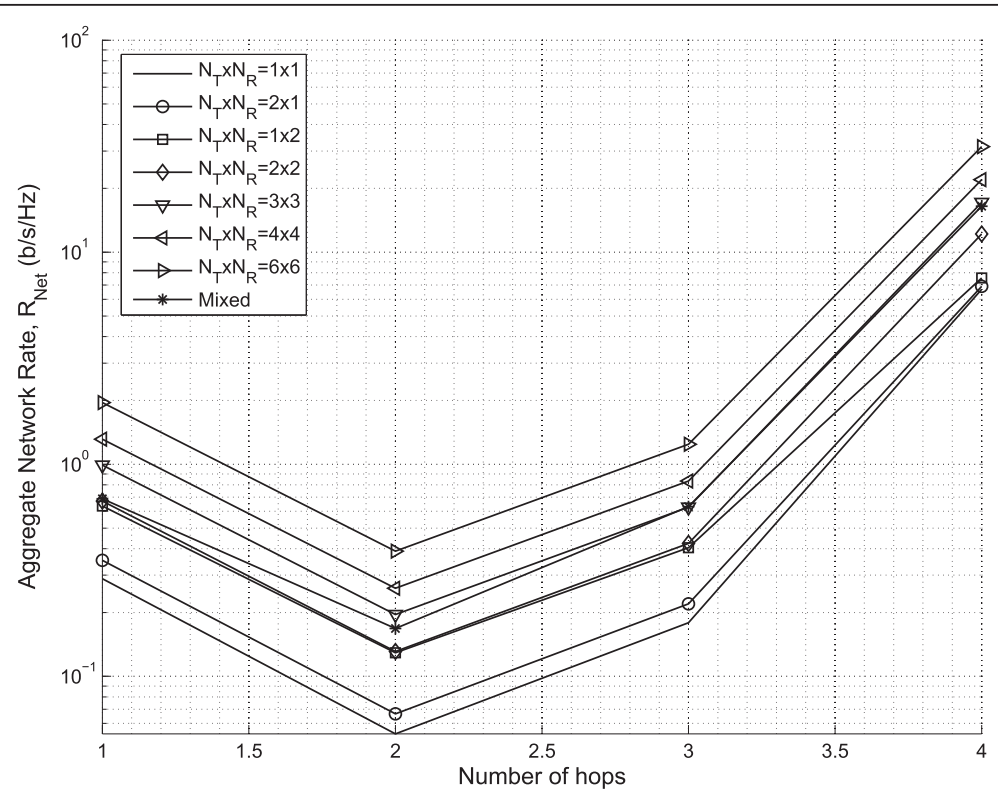

(a) ManhattanMH MIMO cell.

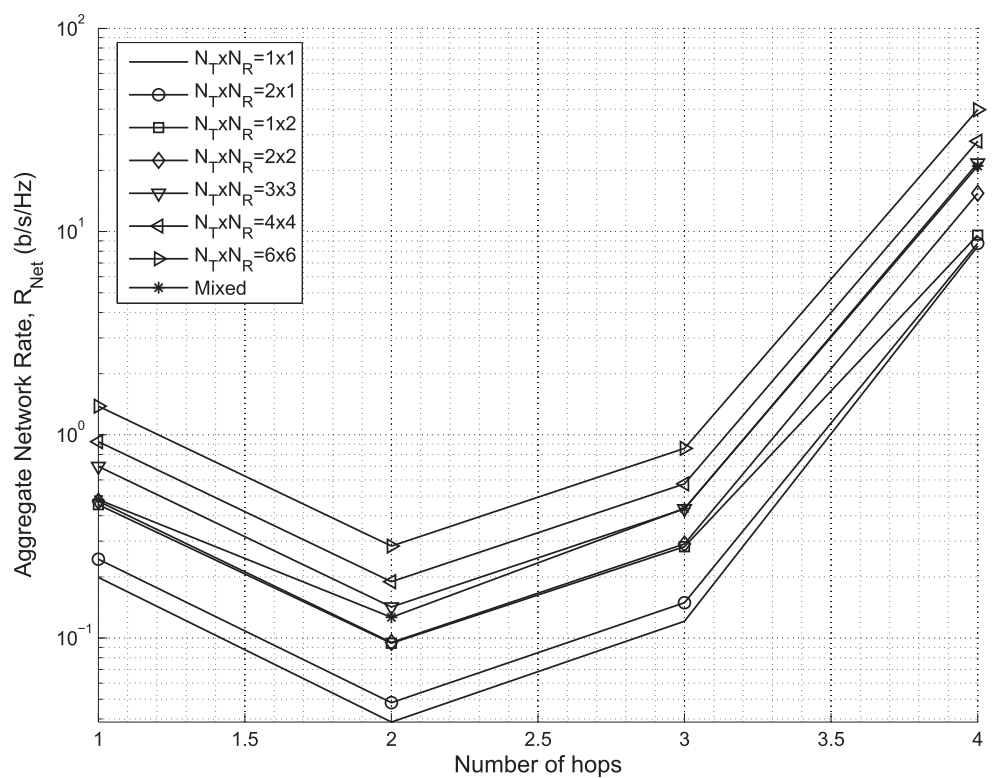

(b) Hexagonal MH MIMO cell.

Figure 13 Aggregate network rate for 1,000 m radius MH MIMO cells. a Manhattan MH MIMO cell. b Hexagonal MH MIMO cell.

received signal-while MIMO tries to make the best of a bad situation by collecting and making best use of randomly scattered signals. Consider the ultimate MH system, in which there are an infinite number of relays spaced at zero distance. The signal received at the end destination at any distance from the sender would be perfect, but the cost of relay placement would be infinite, the delay long, and the algorithms and signaling overhead for routing prohibitively complicated. Hence, a sensible application of MIMO with MH relaying in a 
cellular system may exploit the following approaches.

- Add just enough relays to achieve LOS and low path loss between stations. The resulting small subcells enable higher spectral efficiency per unit area $\left(\mathrm{b} / \mathrm{s} / \mathrm{Hz} / \mathrm{km}^{2}\right)$.

- Use universal frequency reuse among the cells to increase spectral efficiency per unit area.

- Use spatial reuse scheduling among subcells throughout the cell in order to increase spectral efficiency per unit area.

- Beamforming with multiple antennas at the transmit side may reduce co-channel interference.

- Multiple antennas at the receiver will provide array gain.

\section{Conclusions}

We have assembled a realistic model for MH MIMO in a cellular system. This model was used to determine the network capacity and investigate the tradeoffs associated with the combination of $\mathrm{MH}$ relaying and MIMO techniques. MIMO spatial multiplexing can provide great gains in capacity, but only when rich scattering occurs, as is the case when the channel is NLOS. Multihop relaying provides great advantage by relaying around obstacles, reducing the path loss by creating LOS conditions, and enabling spatial reuse of spectrum. We have shown that there is some tradeoff in using these methods simultaneously, but by understanding the nature of this tradeoff in a typical cellular system, we can leverage the benefits of both MH relaying and MIMO. MH relaying can drastically increase SINR, but it still suffers from co-channel interference from neighboring uncoordinated cells. It is expected that network MIMO techniques, in which BSs in different cells coordinate their transmissions, may be used in conjunction with $\mathrm{MH}$ relaying. This is the subject of our current work.

\section{Endnotes}

Endnote A. We use equal power allocation in our work in which all transmit antennas transmit with equal power. This is simpler and more realistic since knowledge of the channel at the transmitter is not needed. With such knowledge, the use of waterfilling on each hop can increase the hop rates, but this will not change any fundamental conclusions.

\section{Acknowledgements}

This work was supported by funding from the Natural Sciences and Engineering Research Council (NSERC) of Canada, TRLabs, Rohit Sharma Professorship, TELUS Communications, and Engineers Canada. The work was presented in part at the 10th International Symposium on Wireless Personal Multimedia Communications (WPMC07), Jaipur, India, 3 - 6 Dec 2007, and at GLOBECOM 2008, New Orleans, LA, USA, 30 Nov - 4 Dec 2008.

\section{Author details}

${ }^{1}$ Department of Electrical and Computer Engineering, University of Alberta, Edmonton, AB, T6G 2V4, Canada ${ }^{2}$ TRLabs, Edmonton, AB, Canada

\section{Competing interests}

The authors declare that they have no competing interests.

Received: 2 September 2010 Accepted: 18 August 2011

Published: 18 August 2011

\section{References}

1. IE Telatar, Capacity of multi-antenna Gaussian channels. Eur Trans Tel. 10(6), 585-596 (1999). doi:10.1002/ett.4460100604

2. GJ Foschini, MJ Gans, On limits of wireless communications in a fading environment when using multiple antennas. Wirel Pers Commun. 6(3), 311-335 (1998). doi:10.1023/A:1008889222784

3. DC Schultz, B Walke, R Pabst, T Irnich, Fixed and planned relay based radio network deployment concepts, in Proc 10th Wireless World Research Forum, (WWRF. New York, USA: The Institution of Electrical Engineers, October 2003). ISBN: 0-85296-753-5

4. T Irnich, DC Schultz, R Pabst, P Wienert, Capacity of a relaying infrastructure for broadband radio coverage of urban areas, in Proc 10th WWRF meeting, New York, (Wireless World Research Forum (WWRF), 2003)

5. H Bolukbasi, H Yanikomeroglu, D Falconer, S Periyalwar, On the capacity of cellular fixed relay networks, in Proc Canadian Conf on Elect and Comp Eng. 4, 2217-2220 (2004)

6. A Urie, The future of mobile networking will be small cells, in Alcatel-Lucent, Tech Rep, Presentation to IEEE IOFC Workshop, PIMRC'09, Tokyo, Japan (September 2009)

7. H Yanikomeroglu, Fixed and mobile relaying technologies for cellular networks, in Proc Second Workshop on Applications and Services in Wireless Networks (ASWN'02), Paris, France, pp. 75-81 (July 2002)

8. R Schoenen, B Walke, On PHY and MAC performance of 3G-LTE in a multihop cellular environment, in Proc WiCom, pp. 926-929 (September 2007)

9. O Oyman, NJ Laneman, S Sandhu, Multihop relaying for broadband wireless mesh networks: From theory to practice. IEEE Commun Mag. 45(11), 116-122 (2007)

10. SW Peters, R Heath Jr, The future of WiMAX: Multihop relaying with IEEE 802.16j. IEEE Commun Mag. 47(1), 104-111 (2009)

11. SW Peters, AY Panah, KT Truong, RW Heath, Relay architectures for 3GPP LTE-Advanced. EURASIP J Wirel Commun Netw 1-14 (March 2009)

12. J Sydir, R Taori, An evolved cellular system architecture incorporating relay stations-[WiMAX update]. IEEE Commun Mag. 47(6), 115-121 (2009)

13. Requirements, evaluation criteria and submission templates for the development of IMT-Advanced. ITU-R, Tech Rep, ITU-R M.2133 (2008)

14. Requirements related to technical performance for IMT-Advanced radio interface(s). ITU-R, Tech Rep, ITU-R M.2134 (2008)

15. Guidelines for evaluation of radio interface technologies for IMT-Advanced. ITU-R, Tech Rep (2008). ITU-R M.2135

16. IEEE Standard for Local and Metropolitan Area Networks-Part 16. Air Interface for Broadband Wireless Access Systems, Amendment 1- Multiple Relay Specification (June 2009). IEEE Std. 802.16j-2009

17. IEEE $802.16 \mathrm{~m}$ system description document. IEEE, Tech Rep, 802.16m-09/ 0034r2 (June 2009)

18. 3rd generation partnership project; technical specification group radio access network; feasibility study for further advancements for E-UTRA (LTEAdvanced) (release 9). 3GPP, Tech Rep, 3GPP TR 36.912 V9.0.0 (September 2009)

19. S Parkvall, E Dahlman, A Furuskar, Y Jading, M Olsson, S Wanstedt, K Zangi, LTE-Advanced-Evolving LTE towards IMT-Advanced, in Proc VTC Fall, pp. 1-5 (September 2008)

20. H Bölcskei, RU Nabar, O Oyman, AJ Paulraj, Capacity scaling laws in MIMO relay networks. IEEE Trans Wireless Commun. 5(6), 1433-1444 (June 2006)

21. IEEE Standard for Local and Metropolitan Area Networks-Part 16. Air Interface for Broadband Wireless Access Systems (May 2009). IEEE Std. 802.16-2009

22. Universal mobile telecommunications system (UMTS); spatial channel model for multiple input multiple output (MIMO) simulations. ETSI, Tech Rep (September 2003). 3GPP TR 25.996 Version 6.1.0 Release 6

23. K Jacobson, WA Krzymień, Multi-hop relaying and multiple antenna techniques-performance trade-offs in cellular systems. In Proc WPMC, Jaipur, India (December 2007) 
24. K Jacobson, WA Krzymień, Multi-hop relaying and MIMO techniques in cellular systems-throughput achievable on Rayleigh/Ricean channels, in Proc Globecom, (New Orleans, Louisiana, November 2008)

25. K Jacobson, WA Krzymień, Realistic throughput of cellular multi-hop relay networks with spatial reuse, in Proc WPMC-2006, (San Diego, USA, 2006), pp. 966-970

26. K Jacobson, WA Krzymień, Cell dimensioning and network throughput in cellular multi-hop relay networks, in Proc VTC2006-Fall, (Montreal, Canada, September 2006)

27. K Jacobson, WA Krzymień, System design and throughput analysis for multi-hop relaying in cellular systems. IEEE Trans Veh Technol. 58(8), 4514-4528 (2009)

28. A Paulraj, R Nabar, D Gore, in Introduction to Space-Time Wireless Communications, (Cambridge, UK: Cambridge University Press, 2003)

29. J Salo, F Mikas, P Vainikainen, An upper bound on the ergodic mutual information of Rician fading MIMO channels. IEEE Trans Wireless Commun. 5(6), 1415-1421 (2006)

30. RA Horn, CR Johnson, in Matrix analysis, (New York, NY, USA: Cambridge University Press, 1985)

31. V Erceg., et al, Channel models for fixed wireless applications. IEEE, Tech Rep, IEEE-C802.16.3c-01/29r4 (July 2001)

32. DS Baum, et al, An interim channel model for beyond-3G systems: extending the 3GPP spatial channel model (SCM), in Proc VTC2005-Spring, (Stockholm, Sweden, 2005), pp. 3132-3136

33. LJ Greenstein, S Ghassemzadeh, V Erceg, DG Michelson, Ricean K-factors in narrowband fixed wireless channels, in Proc WPMC-1999 (September 1999)

34. JN Laneman, GW Wornell, Distributed space-time-coded protocols for exploiting cooperative diversity in wireless networks. IEEE Trans Inform Theory. 49(10), 2415-2425 (2003). doi:10.1109/TIT.2003.817829

doi:10.1186/1687-1499-2011-65

Cite this article as: Jacobson and Krzymień: Multihop relaying and multiple antenna techniques: performance trade-offs in cellular systems. EURASIP Journal on Wireless Communications and Networking 2011 2011:65.

\section{Submit your manuscript to a SpringerOpen ${ }^{\mathcal{O}}$ journal and benefit from:}

- Convenient online submission

- Rigorous peer review

- Immediate publication on acceptance

- Open access: articles freely available online

- High visibility within the field

- Retaining the copyright to your article

Submit your next manuscript at $\gg$ springeropen.com 